Revista Brasileira de Meteorologia, v.29, n.3, 351 - 366, 2014

http://dx.doi.org/10.1590/0102-778620100275

\title{
PREVISÃO DE TEMPO POR CONJUNTOS PARA A REGIÃO NORDESTE DO BRASIL
}

\section{CLEITON DA SILVA SILVEIRA ${ }^{1}$; MARIANE MENDES COUTINHO ${ }^{2}$; ALEXANDRE ARAUJO COSTA $^{3}$; PAULO HENRIQUE SANTIAGO DE MARIA ${ }^{4}$}

\author{
${ }^{1}$ Universidade Federal do Ceará, Departamento de Engenharia Hidráulica e Ambiental (UFC/DEHA), \\ Fortaleza, CE, Brasil \\ ${ }^{2}$ Instituto de Aeronáutica e Espaço, Departamento de Ciência e Tecnologia Aeroespacial (IAE/CTA), São \\ José dos Campos, SP, Brasil \\ ${ }^{3}$ Universidade Estadual do Ceará (UECE), Mestrado em Ciências Físicas Aplicada, Fortaleza, CE, Brasil \\ ${ }^{4}$ Instituto Nacional de Pesquisas Espaciais, Centro de Previsão de Tempo e Estudos Climáticos (INPE/ \\ CPTEC), Cachoeira Paulista, SP, Brasil
}

cleitonsilveira16@yahoo.com.br,mmctrue@gmail.com, alexandrearaujoc@gmail.com,paulohsm@gmail.com

Recebido Dezembro de 2010 - Aceito Novembro de 2013

\begin{abstract}
RESUMO
A técnica de previsão de tempo por conjuntos ("ensemble") é implementada para a região Nordeste do Brasil utilizando-se o modelo regional RAMS, inicializado com dados do modelo de circulação geral atmosférico (MCGA) do CPTEC. Os métodos empregados para geração dos conjuntos de previsão consistem na utilização de diferentes parametrizações físicas e esquemas de relaxamento newtoniano para cada rodada do modelo (ENSFI), e na perturbação das condições iniciais (ENSCI e ENSCIFRONT). Esses métodos são avaliados utilizando-se análises do MCGA e dados das plataformas de coletas de dados localizadas no estado do Ceará. As perturbações nas condições iniciais para o ENSCI e ENSCI-FRONT são geradas com base no método "lagged-average forescasting" a partir de execuções anteriores do modelo válidas para o horário e região de estudo, e incluídas nas componentes horizontais do vento de modo a apresentarem um desvio padrão de $5 \mathrm{~m} \cdot \mathrm{s}^{-1}$. O ENSCI-FRONT inclui perturbações, também nas fronteiras do modelo regional para lidar com uma rápida diminuição do espalhamento dos membros com o prazo de previsão encontrada com perturbações apenas nas condições iniciais. Os resultados mostram que as médias das previsões para o ENSCI-FRONT e ENSFI apresentam melhores resultados do que a previsão não-perturbada para a maioria das grandezas físicas analisadas.

Palavras-chave: Previsão por ensemble, modelos numéricos de tempo, verificação.
\end{abstract}

\begin{abstract}
ENSEMBLE PREDICTION SYSTEM FOR NORTHEAST BRAZIL Ensemble prediction is implemented for Northeast Brazil using RAMS mesoscale model, initialized with data from the CPTEC atmospheric general circulation model (AGCM). Methods for the ensemble generation consist of using different physical parametrizations and nudging timescales (Newtonian relaxation) for each model run (ENSFI), or perturbing the initial conditions (ENSCI and ENSCI-FRONT). These methods are evaluated against the AGCM analyses and data of automatic meteorological stations located in Ceará State. Perturbations in the initial conditions for ENSCI and ENSCI-FRONT are based on the method "lagged-average forecasting", which takes previous model executions valid for the time and region of interest, and are included in the horizontal components of the wind, imposing a standard deviation of $5 \mathrm{~m} . \mathrm{s}^{-1}$. ENSCI-FRONT also includes perturbations in the regional model lateral boundary conditions to deal with a rapid decrease of the ensemble dispersion with the forecast range found when perturbations are only included in the initial conditions. The mean of the perturbed forecasts obtained with ENSCI-FRONT or ENSFI give better results than the unperturbed forecast in most cases.
\end{abstract}

Keywords: Ensemble forecasts; Numerical models; Verification. 


\section{INTRODUÇÃO}

As previsões numéricas de tempo fornecem informações importantes para diversos setores da sociedade, principalmente para aqueles sensíveis a essa variável atmosférica, como a agricultura, recursos hídricos, transporte, energia hidráulica etc. Além disso, a possibilidade de prever eventos extremos permite que decisões possam ser tomadas para que os impactos de tais eventos sejam minimizados, reduzindo assim o grau de vulnerabilidade de uma determinada região (Silveira et al., 2011)

A previsão de fenômenos de mesoescala ainda é um dos principais desafios da previsão numérica de tempo. Apesar dos grandes avanços já obtidos nas previsões de tempo e clima, muito ainda precisa ser feito para a melhoria dos seus índices de acertos. Algumas das principais linhas neste sentido são as previsões por conjuntos (Hou et. al., 2001) e o desenvolvimento e melhoria de parametrizações para aprimorar a representação de processos físicos em escala de subgrade.

Os modelos atmosféricos determinísticos apresentam maiores deficiências quando simulam médios ou longos períodos, pois o crescimento dos erros nas condições iniciais, devido ao comportamento caótico da atmosfera (Lorenz, 1963 e 1965), pode levar a previsões diferindo bastante da realidade. A técnica de previsão por conjunto (ensemble) surge como uma forma de tratar erros cometidos na geração das condições iniciais da atmosfera e também os causados pelas deficiências do modelo, avançando no sentido de dar um caráter probabilístico à previsão, buscando ampliar o prazo de previsão dos modelos e obter melhores previsões numéricas de tempo.

Essa técnica considera uma série de condições iniciais distintas (Leith, 1974; Hoffman e Kalnay, 1983), ou um conjunto de situações com o modelo ligeiramente modificado (Stensurd et al., 1998), ou ainda, previsões a partir de um conjunto de modelos distintos (Krishnamurti e Zhang, 1999; Hou et al., 2001; Silveira et al., 2011).

$\mathrm{Na}$ tentativa de minimizar os efeitos das incertezas nas condições iniciais e fronteiras várias técnicas de previsão por ensemble são amplamente empregadas em diversos centros de meteorologia, como o European Centre For Medium Range Weather Forecast (ECMWF) (Buizza e Palmer, 1995) e National Centers for Environmental Prediction (NCEP) (Toth e Kalnay, 1993), que implementaram operacionalmente a previsão por conjunto em 1992. Já no Brasil esta ferramenta foi implementada em outubro de 2001 no CPTEC/INPE, com origem na dissertação de mestrado de Coutinho (1999), onde foi mostrado que a previsão por conjuntos de um modelo global atmosférico poderia trazer benefícios para a previsão do tempo.

Algumas técnicas de previsão por conjunto estão ligadas, principalmente, ao tratamento de incertezas associadas às aproximações físicas dos modelos. Por exemplo, a utilização de diferentes esquemas de parametrização (Silveira, 2008; Hou et.al, 2001; Bustamante e Chou, 2006) ou diferentes modelos para criar um conjunto de previsões (Silveira et al., 2011).

Neste sentido, o presente estudo tem como objetivo avaliar, por meio da estimativa de índices estatísticos, a possibilidade de se utilizar as técnicas de ensemble físico e ensemble da condição inicial em simulações do Regional Atmospheric Modeling System (RAMS), para realização de previsões de tempo sobre a região Nordeste do Brasil com ênfase sobre o estado do Ceará.

\section{MATERIAIS E MÉTODOS}

\subsection{Técnicas usadas para produção dos conjuntos}

No presente estudo foi utilizado o modelo Regional Atmospheric Modeling System (RAMS 6.0, Cotton et al., 2003), que é um modelo de circulação regional com prognóstico euleriano, baseado na integração das equações primitivas de conservação do momento linear, massa, energia e água. Utilizou-se uma grade polar estereográfica, com 43 pontos nas duas direções horizontais (latitudinal e longitudinal), espaçamento de $39 \mathrm{~km}$ e 42 níveis verticais sigma, centrada em $5,30^{\circ} \mathrm{S}$, $39,33^{\circ} \mathrm{W}$, cobrindo boa parte do Nordeste Brasileiro, assim como uma porção do Oceano Atlântico, conforme mostra a Figura 1. As condições iniciais e de contorno utilizadas são as análises diárias obtidas do NCEP, para a produção das previsões de tempo no CPTEC, com resolução horizontal T126 e 28 níveis na vertical.

Três conjuntos de previsões são gerados para o período de 14/01/2008 a 18/01/2008: um ensemble físico (ENSFI),

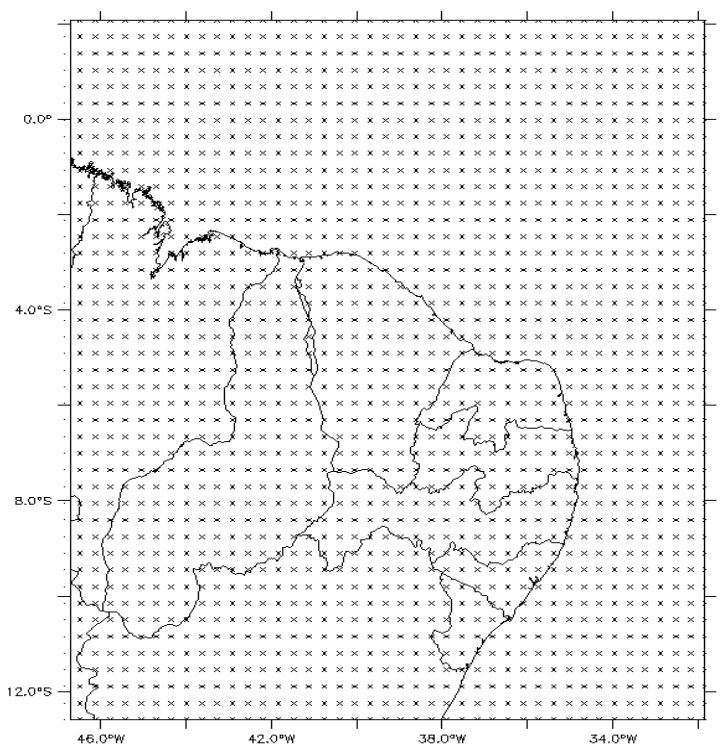

Figura 1 - Grade horizontal utilizada. 
um ensemble de condição inicial (ENSCI) e um ensemble de condição inicial e de fronteira (ENSCI-FRONT). Essas técnicas serão descritas nas seções 2.1.1, 2.1.2 e 2.1.3.

O período simulado encontra-se na transição entre as estações seca e chuvosa. Nos meses de dezembro e janeiro, chamados também de pré-estação, os principais sistemas causadores de chuva na região NEB são: a proximidade das frentes frias e os vórtices ciclônicos de ar superior (Albuquerque et al., 2009). Esse período corresponde também a uma fase de transição do regime local de ventos na região (De Maria et al., 2008).

\subsubsection{Ensemble físico (ENSFI)}

O ENSFI é obtido a partir da utilização de diferentes configurações físicas para cada rodada do modelo (mantendo as mesmas condições iniciais), conforme mostra a Tabela 1. As variações na configuração consistem no uso de diferentes parametrizações e intervalos de "nudging" central, de acordo com as opções a seguir:

- Parametrizações de convecção: Kuo (Tremback, 1990) ou Kain-Fritsch (Kain, 2002).

- Parametrizações de turbulência: Mellor e Yamada (1974) ou Smagorinsky (1963) com deformação anisotrópica.

- Parametrizações de radiação: Harrington (1997) ou Chen e Cotton (1983).

- Nudging central: acionado a cada 12 ou 24 horas. As simulações combinando Smagorinsky e Kain-Fritsch não foram completadas devido a problemas de instabilidade numérica, resultando em um conjunto de 12 membros. As condições iniciais e de contorno são as previsões de tempo do modelo global do CPTEC com resolução horizontal T126 e 28 níveis na vertical

\subsubsection{Ensemble de condição inicial (ENSCI)}

Diversos métodos de previsão por conjunto são gerados a partir da criação das condições iniciais perturbadas (Leith, 1974), estas são geradas tipicamente usando a análise de controle (tradicional) como a melhor estimativa do verdadeiro estado inicial da atmosfera e acrescentando perturbações a esta análise para definir as outras condições iniciais.

Por ser um sistema caótico, a geração das condições iniciais da atmosfera é essencial para a qualidade da previsão resultante do modelo. É importante que o método de geração de condições iniciais utilizado represente bem a incerteza associada ao estado inicial da atmosfera e mantenha, dentro da amplitude do conjunto de previsões dos membros do ensemble, o estado real futuro da atmosfera.

O método para gerar as condições iniciais para o ENSCI e o ENSCI-FRONT baseia-se no "lagged-average forescasting" (Hoffman e Kalnay, 1983; Toth e Kalnay, 1993). Este método consiste em obter previsões a partir de perturbações geradas com base em simulações anteriores do modelo válidas para o horário de interesse. Para este caso, as perturbações são as diferenças ou soma entre cada uma das previsões anteriores válidas para o horário da análise e a análise em consideração. A vantagem deste método é que as perturbações são geradas a partir de previsões reais, influenciadas, portanto, pelo fluxo atmosférico corrente, de modo que as perturbações obtidas estão associadas a erros que crescem no modelo na situação atmosférica considerada. Contudo, esse método tem a desvantagem que as previsões geradas a partir de perturbações mais antigas, em

Tabela 1 - Configurações do modelo usadas no ensemble físico.

\begin{tabular}{ccccc}
\hline \hline $\begin{array}{c}\text { Membro } \\
\text { do ensemble }\end{array}$ & $\begin{array}{c}\text { Esquema } \\
\text { de convecção }\end{array}$ & $\begin{array}{c}\text { Esquema } \\
\text { De turbulência }\end{array}$ & $\begin{array}{c}\text { Esquema } \\
\text { de radiação }\end{array}$ & $\begin{array}{c}\text { Escala de } \\
\text { nudging }\end{array}$ \\
\hline $\begin{array}{c}\text { har-kuo-mellor-24 } \\
\text { har-kuo-mellor-12 }\end{array}$ & Kuo & Mellor \& Yamada & Harrington & 24 horas \\
chen-kuo-mellor-24 & Kuo & Mellor \& Yamada & Harrington & 12 horas \\
chen-kuo-mellor-12 & Kuo & Mellor \& Yamada & Chen \& Cotton & 12 horas \\
har-kuo-sma -24 & Kuo & Smagorinsky & Harrington & 24 horas \\
har-kuo-sma -12 & Kuo & Smagorinsky & Harrington & 12 horas \\
chen-kuo-sma-24 & Kuo & Smagorinsky & Chen \& Cotton & 24 horas \\
chen-kuo-sma -12 & Kuo & Smagorinsky & Chen \& Cotton & 12 horas \\
har-kain-mellor -24 & Kain-Fritsch & Mellor \& Yamada & Harrington & 24 horas \\
har-kain-mellor -12 & Kain-Fritsch & Mellor \& Yamada & Harrington & 12 horas \\
chen-kain-mellor-24 & Kain-Fritsch & Mellor \& Yamada & Chen \& Cotton & 24 horas \\
chen-kain-mellor-12 & Kain-Fritsch & Mellor \& Yamada & Chen \& Cotton & 12 horas \\
\hline
\end{tabular}


geral, apresentam uma amplitude de erro maior em relação aos outros membros com perturbações de horários posteriores (Toth e Kalnay,1993). Uma maneira de minimizar este problema é utilizar pesos diferentes para cada membro do conjunto.

Para estes conjuntos o membro de controle será a previsão inicializada a partir da análise do modelo global do CPTEC sem perturbação, combinando as parametrizações de Kuo, Mellor e Yamada e Harrington com "nudging" central a cada 24 horas.

A condição inicial para cada um dos demais membros é obtida a partir de perturbações nas componentes horizontais do vento em todos os níveis verticais, associadas à diferença entre uma previsão anterior do modelo (com prazo de 24 , 48 ou 72 h) válida para o horário da análise e a análise em consideração, conforme Figura 2. Essas perturbações são reescalonadas utilizando um desvio padrão de $5 \mathrm{~m} / \mathrm{s}$ (de acordo com estimativas dos erros normalmente existentes na análise obtidas do trabalho de Daley e Mayer, 1986) e, então, adicionadas ou subtraídas da análise, resultando em sete membros no conjunto de previsões final (incluindo a previsão de controle), conforme a Tabela 2.

\subsubsection{Ensemble de condição inicial e fronteira (ENSCI- FRONT)}

A geração das condições iniciais do ENSCI-FRONT é idêntica à feita para o ENSCI, ou seja, para uma mesma data e horário as condições iniciais são as mesmas para os dois casos. A diferença é que para o ENSCI-FRONT a fronteira também é perturbada com base no método "lagged-average forescasting" (Hoffman e Kalnay, 1983), e também a partir de previsões do modelo global do CPTEC.
Para previsão do tempo em escala regional, estes dados alimentam a fronteira lateral do modelo de área limitada a cada seis horas de integração e o esquema de relaxamento newtoniano (nudging) faz com que a solução do modelo regional tenda para esses dados.

A preparação das condições iniciais é feita de acordo com os passos descritos na 2.1.2. Para as condições de fronteira segue-se um procedimento semelhante, a fronteira é perturbada a cada $6 \mathrm{~h}$ a partir da diferença entre as previsões do modelo global inicializadas em diferentes horários com análise.

A perturbação na fronteira, portanto, é gerada usando a previsão do modelo utilizada para perturbar o membro de controle. Para a previsão de $12 \mathrm{~h}$ do membro soma pert. $72 \mathrm{~h}$ (conforme Tabela 2), por exemplo, a perturbação se dá através da previsão de 12 h da rodada inicializada no dia 11/01/2008. Está perturbação é inserida na fronteira do citado membro no dia 14/01/2008 às $12 \mathrm{~h}$.

Neste caso o ensemble ENSCI-FRONT possui sete membros, dos quais um é gerado sem perturbação (idêntico ao controle em ENSCI) e os outros seis são alterados através da soma e subtração de perturbações nas coordenadas do vento, de maneira análoga à descrita na Tabela 2.

\subsection{Dados observados usados para a avaliação dos conjuntos e período simulado}

O período simulado para cada uma das técnicas é de 96 horas a partir das 0000UTC do dia 14 de janeiro de 2008.

Para a avaliação dos conjuntos apresentados neste trabalho foram usadas as análises do modelo global do CPTEC com resolução horizontal T126 e 28 níveis na vertical. Além

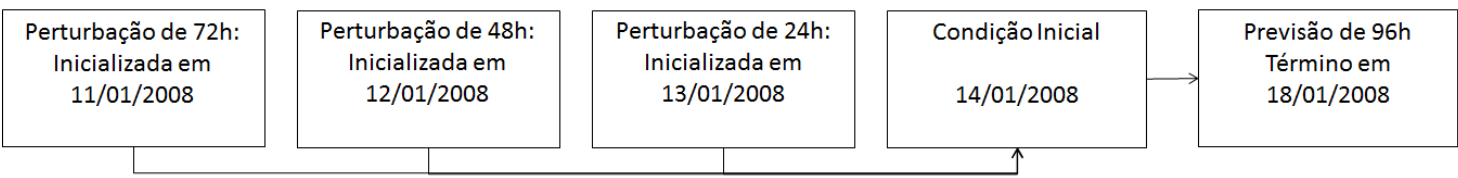

Figura 2 - Período simulado e Análises usadas para geração das perturbações dos conjuntos ENSCI e ENSCI-FRONT.

Tabela 2 - Membros do ensemble de condição inicial.

\begin{tabular}{cc}
\hline \hline $\begin{array}{c}\text { Membros do } \\
\text { Ensemble }\end{array}$ & Condições Iniciais \\
\hline não pert. & \multicolumn{1}{c}{ Análise não perturbada } \\
soma pert. de $24 \mathrm{~h}$ & Análise+Perturbação associada à previsão de 24 horas \\
soma pert. de $48 \mathrm{~h}$ & Analise+Perturbação associada à previsão de 48 horas \\
soma pert. de $72 \mathrm{~h}$ & Analise+Perturbação associada à previsão de 72 horas \\
subtrai pert. de $24 \mathrm{~h}$ & Analise-Perturbação associada à previsão de 24 horas \\
subtrai pert. de $48 \mathrm{~h}$ & Analise-Perturbação associada à previsão de 48 horas \\
subtrai pert. de $72 \mathrm{~h}$ & Analise-Perturbação associada à previsão de 72 horas \\
\hline
\end{tabular}


disso, foram selecionadas informações de 11 Plataformas de Coleta de Dados (PCDs) da Fundação Cearense de Meteorologia e Recursos Hídricos (FUNCEME), distribuídas por todo Ceará, conforme mostra a Tabela 3.

\section{3 Índices estatísticos}

As medidas estatísticas utilizadas, cujas definições estão indicadas a seguir, são: erro de viés (BIAS Error), raiz do erro quadrático médio (RMSE), correlação (CORREL) e erro quadrático médio dos desvios com eliminação do viés (URMS).

O erro de viés mede a tendência do modelo para superestimar ou subestimar uma variável, e é definido por

$$
\text { Viés }=\frac{1}{n} \sum_{i=1}^{n}(P-A)
$$

onde $\mathrm{n}$ é o número total de observações/previsões, e o índice P e A indicam previsão e análise verificada e/ ou os valores observados pelas PCDs, respectivamente. Um valor positivo do erro de viés indica uma predisposição do modelo em superestimar uma variável particular. Reciprocamente, um valor negativo do erro de viés implica numa subestimação do modelo na avaliação de uma variável.

A raiz do erro quadrático médio (RMSE, Root Mean Square Error) é a raiz quadrada da média das diferenças individuais quadráticas entre a previsão e as observações e é definida pela equação

$$
R M S E=\sqrt{\frac{1}{n} \sum_{i=1}^{n}(P-A)^{2}}
$$

Essa medida retém as unidades físicas da variável prevista e mede o erro típico da previsão do modelo. Valores grandes do RMSE representam grandes erros nos campos previstos, e valores próximos de zero indicam uma previsão quase perfeita. Elevando ao quadrado o termo da diferença, o RMSE tende a dar maior peso às grandes discrepâncias entre os campos observados e previstos.

A correlação pode assumir valores entre -1 e 1 que indicam, respectivamente, perfeita anticorrelação e perfeita correlação, havendo ainda a total ausência de correlação verificada com um resultado igual a zero. Este índice tem a capacidade de detectar correspondência de fase entre as séries, sendo, por construção, insensível ao viés.

$$
\text { CORREL }=\frac{\sum_{i=1}^{n}\left(A_{i}-\bar{A}\right)\left(P_{i}-\bar{P}\right)}{\sqrt{\sum_{i=1}^{n}\left(P_{i}-\bar{P}\right)^{2} \sum_{i=1}^{n}\left(A_{i}-\bar{A}\right)^{2}}}
$$

Geralmente, as possíveis causas para a ocorrência de erros sistemáticos nas previsões são: a formulação inadequada
Tabela 3-PCDs usadas para avaliação do vento em 10 metros de altura.

\begin{tabular}{ccc}
\hline \hline PCD & Latitude & Longitude \\
\hline Acopiara & $06^{\circ} 05^{\prime} 53.6^{\prime \prime}$ & $39^{\circ} 12^{\prime} 36.5^{\prime \prime}$ \\
Beberibe & $04^{\circ} 14^{\prime} 29,9^{\prime \prime}$ & $38^{\circ} 12^{\prime} 05,3^{\prime \prime}$ \\
Crato & $07^{\circ} 11^{\prime} 46.2^{\prime \prime}$ & $39^{\circ} 30^{\prime} 22.4^{\prime \prime}$ \\
Granja - Gangorra & $0^{\circ} 09^{\prime} 53,2^{\prime \prime}$ & $40^{\circ} 51^{\prime} 40,6^{\prime \prime}$ \\
Ibaretama & $0^{\circ} 49^{\prime} 36,8^{\prime \prime}$ & $38^{\circ} 49^{\prime} 51,0^{\prime \prime}$ \\
Itapipoca - Quandu & $03^{\circ} 34^{\prime} 24.1^{\prime \prime}$ & $39^{\circ} 37^{\prime} 40.0^{\prime \prime}$ \\
Maranguape & $03^{\circ} 59^{\prime} 06,1^{\prime \prime}$ & $38^{\circ} 47^{\prime} 17,5^{\prime \prime}$ \\
Pacajus & $04^{\circ} 11^{\prime} 24,0^{\prime \prime}$ & $38^{\circ} 29^{\prime} 54,2^{\prime \prime}$ \\
Pereiro & $06^{\circ} 02^{\prime} 23.5^{\prime \prime}$ & $38^{\circ} 28^{\prime} 02.0^{\prime \prime}$ \\
Quixadá & $04^{\circ} 59^{\prime} 18,5^{\prime \prime}$ & $39^{\circ} 00^{\prime} 40,2^{\prime \prime}$ \\
Russas & $05^{\circ} 02^{\prime} 30.4^{\prime \prime}$ & $38^{\circ} 07^{\prime} 08.8^{\prime \prime}$ \\
\hline
\end{tabular}

dos modelos, imprecisão causada pela discretização espaçotemporal e imprecisão dos próprios instrumentos usados na coleta dos dados observados. Tais erros podem ser reduzidos com a remoção do viés estatístico, ou seja, quando as medidas de erro são calculadas apenas para os desvios das séries. Seguindo este raciocínio, será apresentado o Erro quadrático médio dos desvios com eliminação do viés (URMS):

$$
U R M S=\sqrt{\sum_{i=1}^{n}\left(P_{i}^{\prime}-A_{i}^{\prime}\right)^{2}}
$$

sendo $\mathrm{A}_{\mathrm{i}}^{\prime}=A_{i}-\bar{A}$ e $\mathrm{P}_{\mathrm{i}}^{\prime}=P_{i}-\bar{P}$.

\section{RESULTADOS E DISCUSSÕES}

\subsection{Descrição sinótica da região Nordeste do Brasil no período simulado}

Na Figura 3 é mostrada a descrição sinótica da região Nordeste do Brasil no período entre 0000UTC de 14 e 0000UTC de 18 de janeiro de 2008.

O padrão de escoamento em altos níveis sobre a região Nordeste do Brasil (NEB) no período estudado é caracterizado, principalmente, por dois eventos: a dissipação de um Vórtice Ciclônico de Altos Níveis (VCAN), inicialmente verificado sobre o Atlântico, e a evolução de um cavado sobre o NEB com eixo orientado no sentido SE-NW. Estes sistemas podem ser identificados na Figura 3a, estando o VCAN aproximadamente em $21^{\circ} \mathrm{S}, 34^{\circ} \mathrm{W}$, a partir do qual se estende o cavado. A partir de 0000UTC de 16 de janeiro de 2008 (Figura 3b), o VCAN não pode mais ser identificado, e a extremidade do eixo do cavado sobre o oceano passa a se localizar mais a SE, afastando-se do continente. $\mathrm{O}$ escoamento neste cavado encontra-se perturbado sobre o NEB, o que favorece a formação de nuvens sobre o oeste da região.

Em superfície (Figura 3c), identifica-se a atuação da Alta Subtropical do Atlântico Sul (SUL), cujo centro varia 


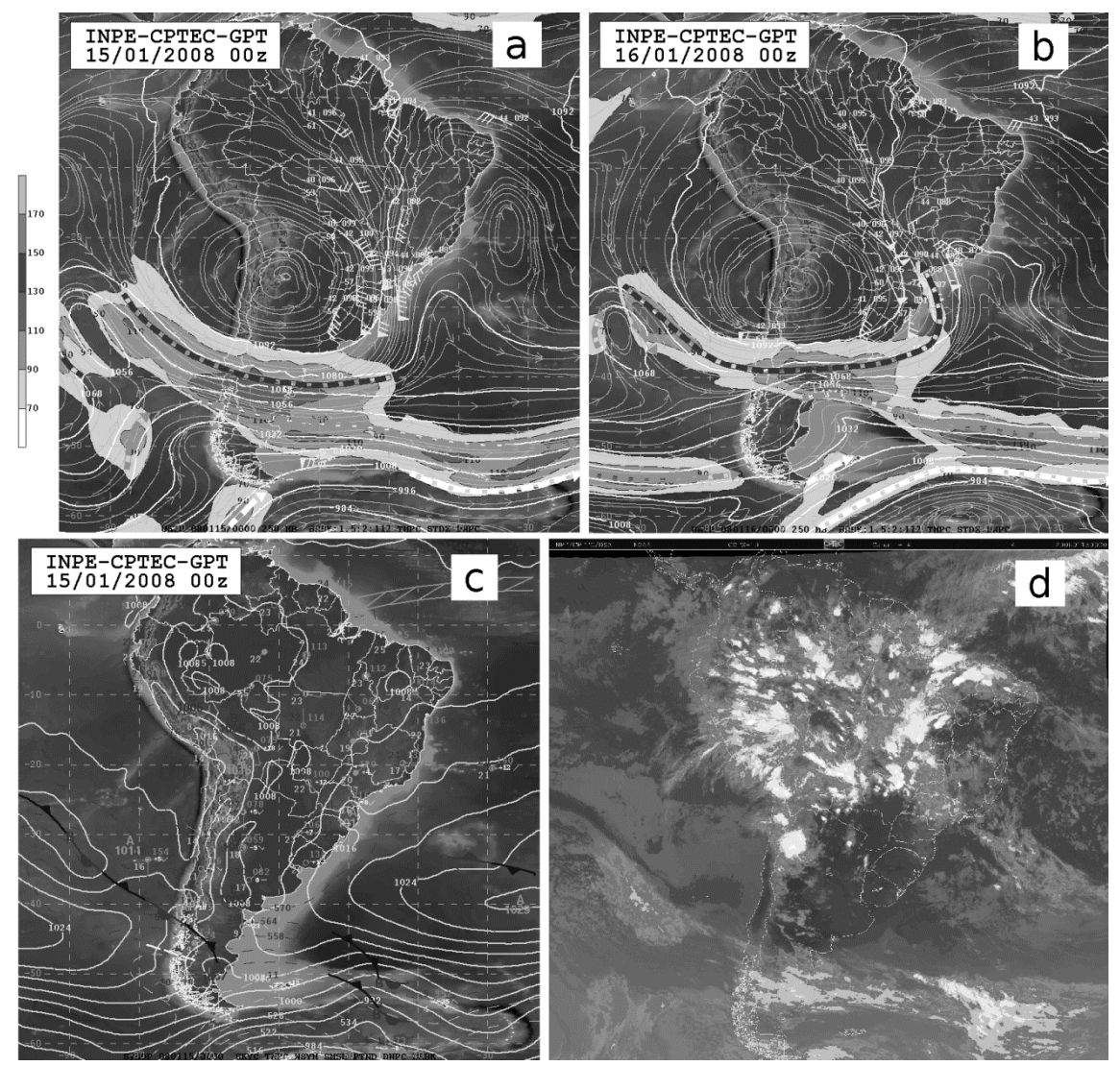

Figura 3 - Carta sinótica (a) de altitude e (b) de superfície, e (c) imagem do canal 4 no infravermelho do satélite GOES-10 para 0000Z de 15/01/2008.

entre as latitudes de $40^{\circ} \mathrm{S}$ e $30^{\circ} \mathrm{S}$ e a longitudes a leste de $35^{\circ} \mathrm{W}$. Este sistema favorece o transporte de umidade do oceano para o continente através do litoral leste do Brasil, para as regiões Nordeste e Sudeste. A posição da Zona de Convergência Intertropical (ZCIT), por sua vez, oscila entre 0 e $7^{\circ} \mathrm{N}$, e influencia o tempo sobre a porção norte do NEB e estados da região Norte, explicando a faixa de nebulosidade que se estende do estado do Rio Grande do Norte ao estado do Pará na Figura 3d.

\subsection{Pressão à superfície}

Na Figura 4 é mostrado o espalhamento (desvio padrão entre os membros do conjunto) da pressão na superfície. Observa-se um espalhamento muito grande do ENSCI nos primeiros passos de tempo do modelo regional, que logo é amortecido, tendendo a zero depois de algumas horas de simulação. Os espalhamentos de outras variáveis não mostrados aqui, como o do vento e da temperatura, apresentam um comportamento similar. Isto é esperado, uma vez que, ao se alimentar os vários membros do modelo regional por informações de grande escala idênticas, correspondendo aos campos de previsão pelo MCGA, a diferença introduzida nas condições iniciais tende a desaparecer. Isso sugere a aplicação de uma perturbação atuante nas fronteiras laterais do modelo, o que é realizado no ENSCI-FRONT. O ENSCI-FRONT apresenta quase sempre um espalhamento maior em relação às outras duas metodologias de ensemble utilizadas para praticamente todas as variáveis e níveis analisados, porém, para as variáveis superficiais e de baixos níveis, provavelmente devido ao uso de diferentes parametrizações físicas, o ENSFI apresenta um espalhamento maior em alguns momentos da simulação, como é o caso do período entre os dias 15 e 16 de janeiro para a pressão à superfície.

Na Figura 5a são destacados os campos de pressão à superfície gerados pelas previsões de 24 horas dos membros do ENSFI, bem como, a média dessas previsões e a correspondente análise. Nota-se que os membros subestimaram a pressão indicada pela análise, com destaque para região litorânea de Alagoas, sobre o Maranhão e no oceano ao sudeste do Rio Grande do Norte.

Nos campos de pressão à superfície dos membros do ENSCI-FRONT para as previsões de 24 horas mostrados na Figura $5 \mathrm{~b}$, nota-se que há uma boa representação do campo verificado pelos membros onde as perturbações foram adicionadas (vide legenda da Figura $5 b$ ), com destaque para a região litorânea de Alagoas e para o Ceará. 


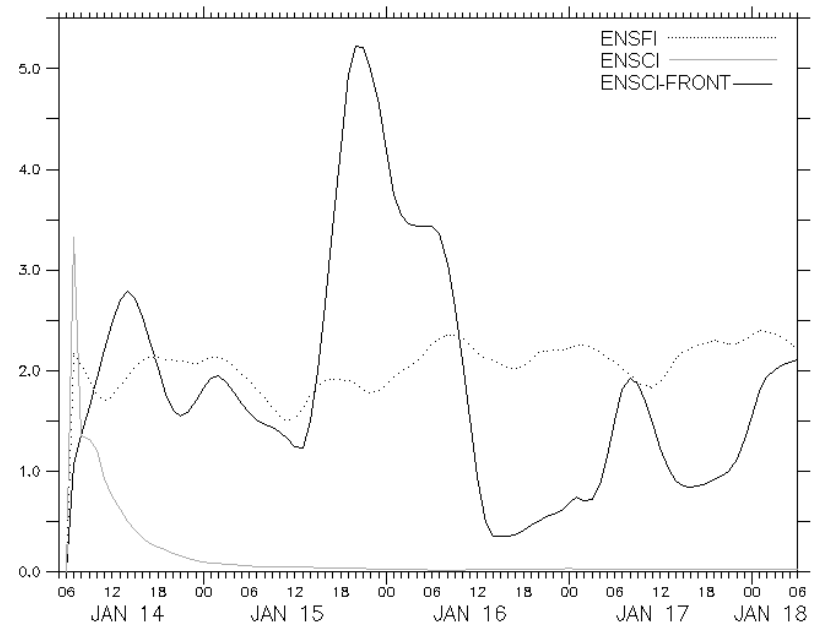

Figura 4 - Espalhamento médio da pressão na superfície (hPa) ao longo das 96 horas de simulação.
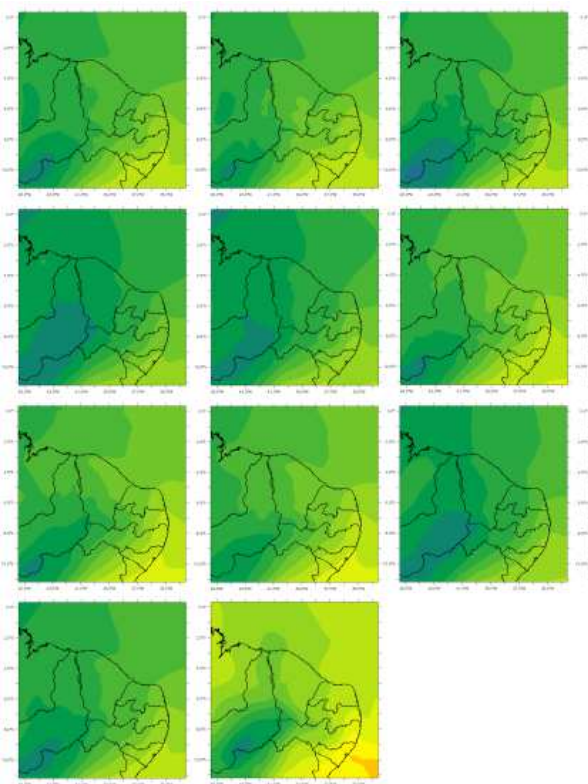

a)
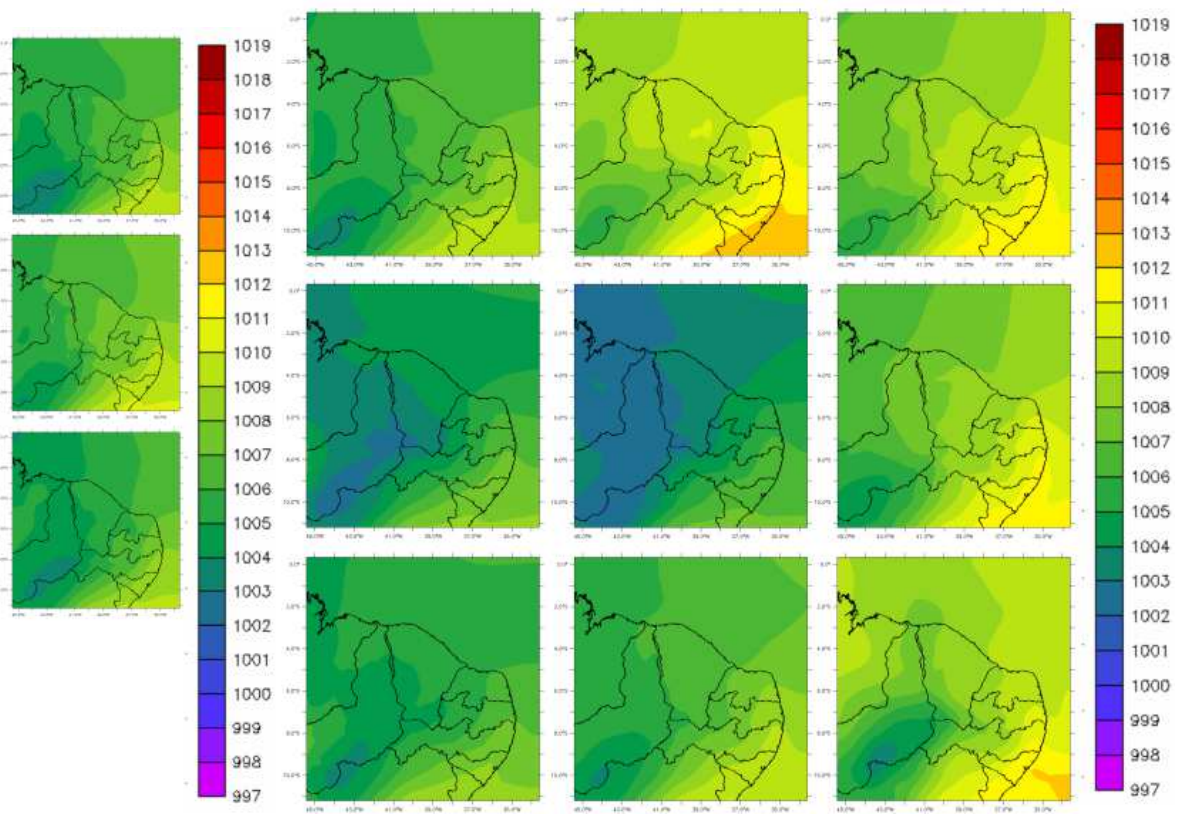

b)

Figura 5 - Campos da previsão de 24 horas para pressão à superfície válidos para as 00h do dia 15/01/2008 do (a) ENSFI na seguinte sequência da esquerda para a direita e de cima para baixo: har-kuo-mellor-24, har-kuo-mellor-12, har-kuo-sma-24, har-kuo-sma-12, har-kain-mellor-24, harkain-mellor-12, chen-kuo-mellor-24, chen-kuo-mellor-12, chen-kuo-sma-24, chen-kuo-sma-12, chen-kain-mellor-24, chen-kain-mellor-12, ensemble médio e análise verificada; e do (b) ENSCI-FRONT na seguinte sequência da esquerda para a direita e de cima para baixo: membro de controle, soma pert.24h, soma pert.48h, soma pert.72h, soma pert.24h, subtrai pert.48h, subtrai pert.72h, ensemble médio e análise verificada.

Na Figura 6 são mostrados os índices estatísticos para pressão à superfície dos membros do ENSFI em relação à análise verificada nos dias 15, 16, 17 e 18 de janeiro de 2008. Há uma tendência dos membros que usam o esquema de nudging central a cada 24 horas apresentarem melhores índices, principalmente para correlação e URMS, em relação aos que usam nudging a cada 12 horas. Além disso, os membros que usam a parametrização de Kuo apresentam melhores previsões em $24 \mathrm{~h}$ e $48 \mathrm{~h}$, do que os membros que usam a parametrização Kain-
Fritsch, tomando por base principalmente o Viés e o RMSE, porém esse comportamento inverte-se nas demais previsões (isto é, 72h e 96h). Na Figura 7 são mostrados os índices estatísticos para pressão à superfície dos membros do ENSCIFRONT, em relação à análise verificada nos dias $15,16,17 \mathrm{e}$ 18 de janeiro de 2008. Os membros do conjunto representaram razoavelmente essa variável, com correlações superiores a 0,6 e URMS em torno de $1 \mathrm{hPa}$ em todo o período simulado. Com relação ao ensemble médio e a previsão de controle, não há uma 

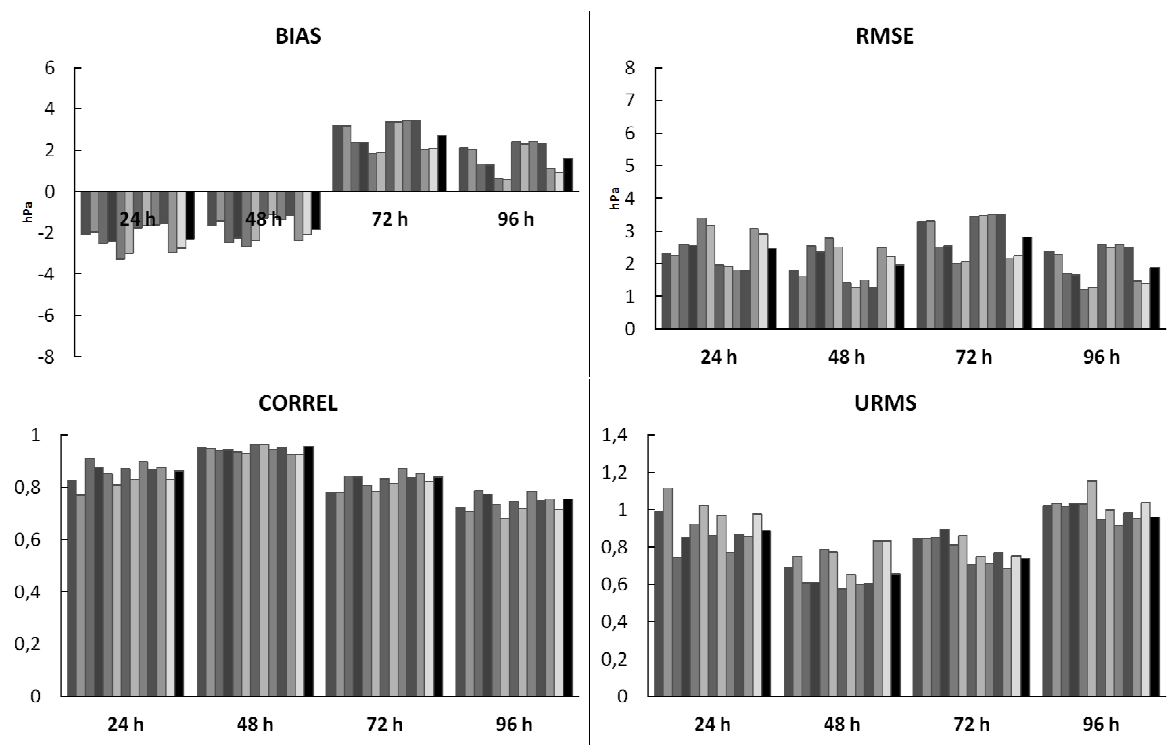

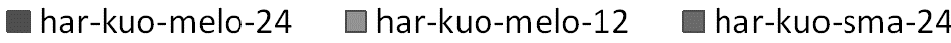

- har-kuo-sma-12 $\square$ har-kain-melo-24 $\square$ har-kain-melo-12

$\square$ chen-kuo-melo-24 $\square$ chen-kuo-melo-12 $\square$ chen-kuo-sma-24

$\square$ chen-kuo-sma-12 $\square$ chen-kain-melo-24 $\square$ chen-kain-melo-12

- ensemble médio

Figura 6 - Índices estatísticos para pressão à superfície para as previsões de 24 h, 48 h, 72 h e 96 h para o ENSFI.
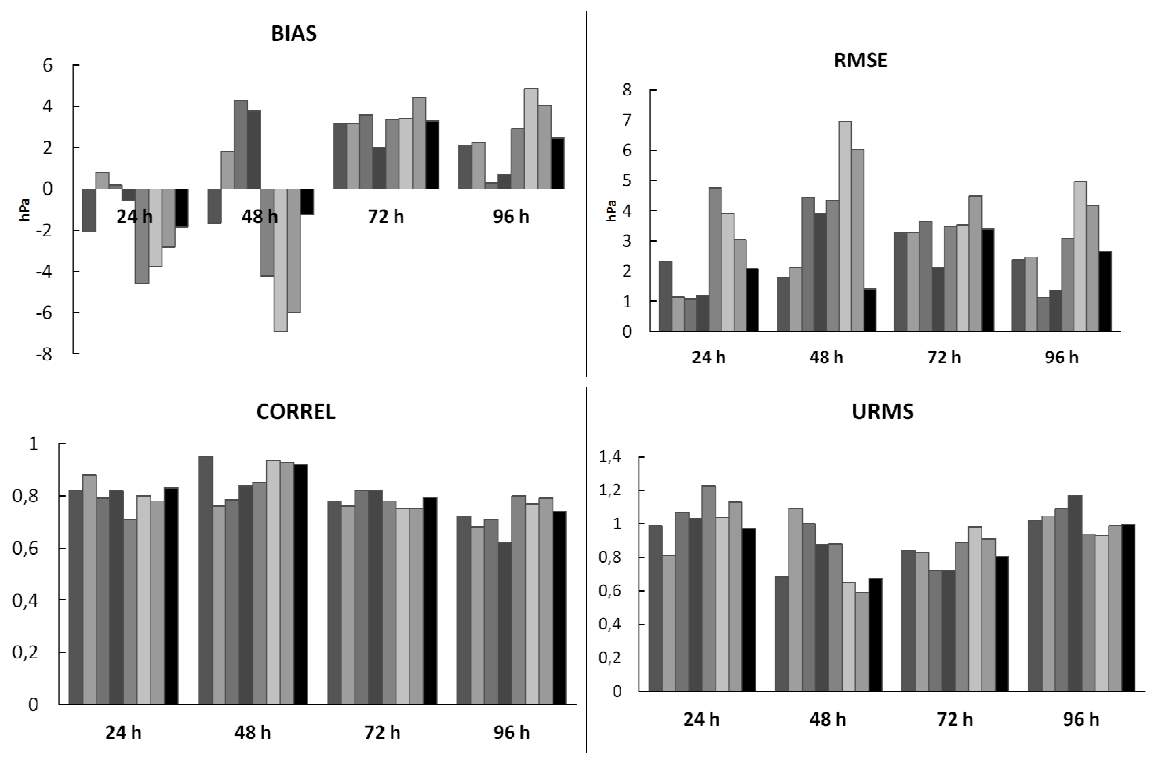

não perturbado $\square$ soma pert.24h $\square$ soma pert. $48 \mathrm{~h}$

soma pert. $72 \mathrm{~h} \quad \square$ subtrai pert. $24 \mathrm{~h} \square$ subtrai pert. $48 \mathrm{~h}$ subtrai pert. $72 \mathrm{~h} \mathbf{\text { ensemble médio }}$

Figura 7 - Índices estatísticos para pressão à superfície para as previsões de 24 h, 48 h, 72 h e 96 h para o ENSCI-FRONT. 
vantagem significativa do ensemble médio para essa variável, porém é importante destacar que em alguns períodos avaliados a melhor previsão é encontrada entre os membros perturbados, principalmente considerando-se o Viés e o RMSE.

As duas técnicas de previsão por ensemble apresentaram correlações bastante significativas para pressão à superfície, indicando uma boa representação do modelo regional para esta variável.

\subsection{Temperatura}

Na Figura 8a são mostrados os campos de temperatura em $925 \mathrm{mb}$ dos membros do ENSFI para a previsão de 24 horas, válida para o dia 15 de janeiro de 2008. Nota-se que a previsão de controle subestimou a temperatura indicada pela análise verificada no oceano ao norte do Maranhão, porém superestimou-a no Ceará. Nota-se também que a previsão dada pelo ensemble médio apresenta grande semelhança com a do membro de controle e a maioria dos membros do ENSFI reproduziram bem a análise verificada.

Na Figura 9b são destacados os campos da temperatura em $925 \mathrm{hpa}$ encontrados pela previsão de 24 horas dos membros do ENSCI-FRONT, válida para o dia 15 de janeiro de 2008. Nota-se que o ensemble médio e membro de controle proporcionaram previsões muito semelhantes nesse nível de pressão, porém a melhor previsão desse ensemble é a do membro "soma pert.24h", com correlações superiores a todos os outros membros em $925 \mathrm{hPa}$, como mostra a Figura 9.

Na Figura 9 são mostrados os índices estatísticos da previsão de 24 horas para temperatura dos membros do ENSFI em relação à análise verificada no dia 15 de janeiro de 2008. O membro "chen-kain-mellor-24" apresentou a melhor previsão em $925 \mathrm{hPa}$. Nota-se também que o ensemble médio apresentou melhores previsões do que o membro de controle (Har-KuoMellor-24:membro com as configurações físicas utilizadas pela previsão de tempo da FUNCEME em janeiro de 2008) em todos os níveis.

Na Figura 10 são mostrados os índices estatísticos da previsão de 24 horas para temperatura dos membros do ENSCIFRONT, em relação à análise verificada no dia 15 de janeiro de 2008. Em geral, o ensemble médio do ENSCI-FRONT apresentou melhores previsões do que o membro de controle, principalmente considerando-se o Viés e o RMSE.

A previsão para $700 \mathrm{hPa}$ apresenta um pequeno RMSE, pequeno URMS e correlação superior a 0,6 para ambas as técnicas. Em $500 \mathrm{hPa}$, as técnicas de previsão por conjunto apresentaram muita dificuldade de representar esta variável, com correlações inferiores a 0,2 . Para $250 \mathrm{hPa}$, os conjuntos de previsões apresentaram correlações entre 0,2 e 0,4 associados a RMSE e URMS bastante elevados, com todos os membros

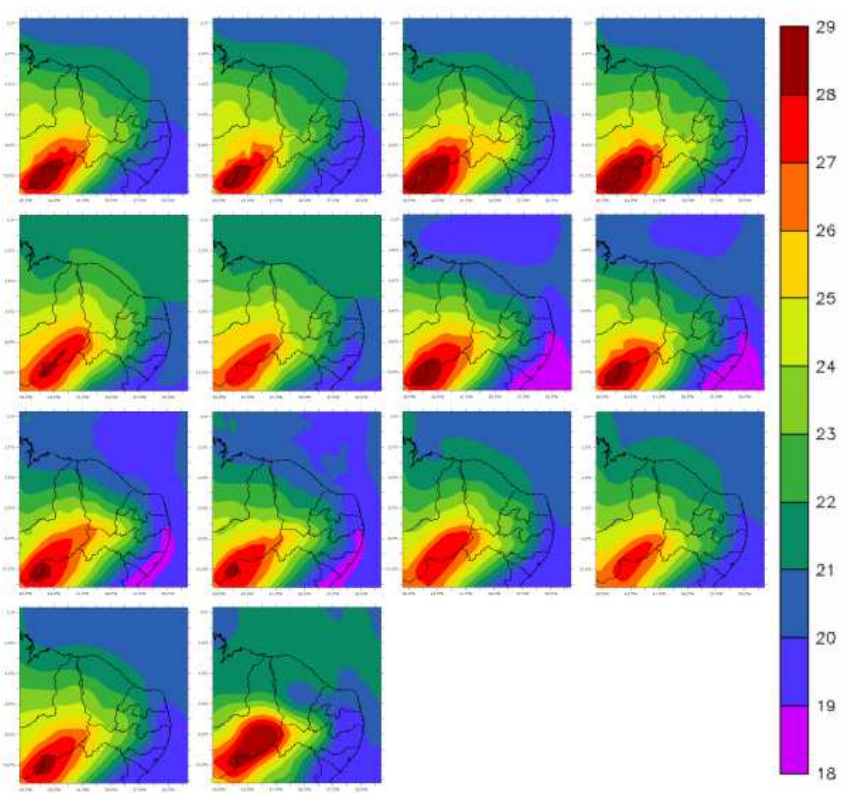

a)
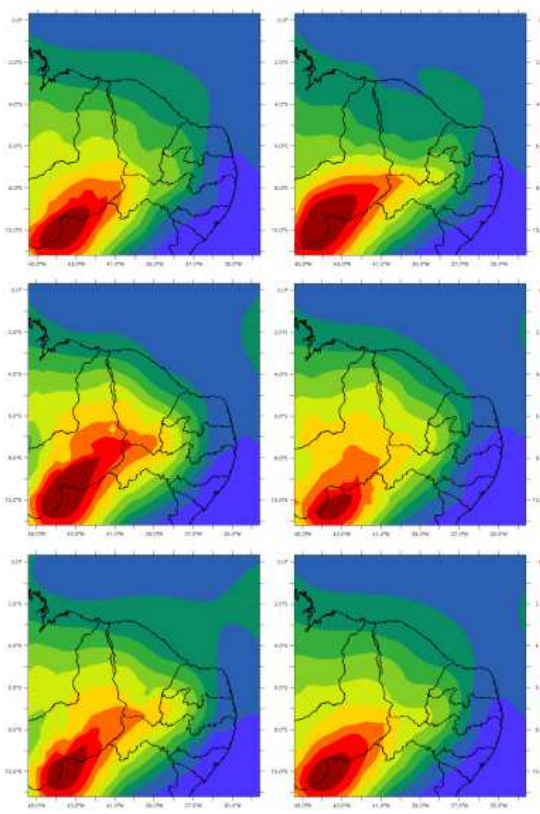

b)

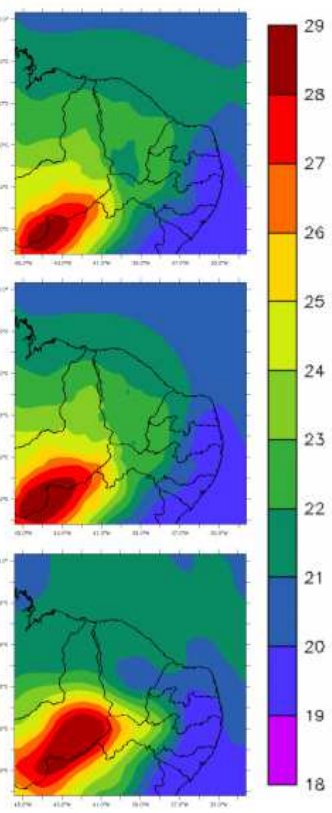

Figura 8 - Campos da previsão de 24 horas para temperatura em 925mb válidos para às 00h do dia 15/01/2008 do (a)ENSFI na seguinte sequência da esquerda para a direita e de cima para baixo har-kuo-mellor-24, har-kuo-mellor-12, har-kuo-sma-24, har-kuo-sma-12, har-kain-mellor-24, harkain-mellor-12, chen-kuo-mellor-24, chen-kuo-mellor-12, chen-kuo-sma-24, chen-kuo-sma-12, chen-kain-mellor-24, chen-kain-mellor-12, ensemble médio e análise verificada; e (b)ENSCI-FRONT na seguinte sequência da esquerda para a direita e de cima para baixo: membro de controle, soma pert. $24 \mathrm{~h}$, soma pert. $48 \mathrm{~h}$, soma pert.72h, soma pert.24h, subtrai pert.48h, subtrai pert.72h, ensemble médio e análise verificada. 
BIAS-24h

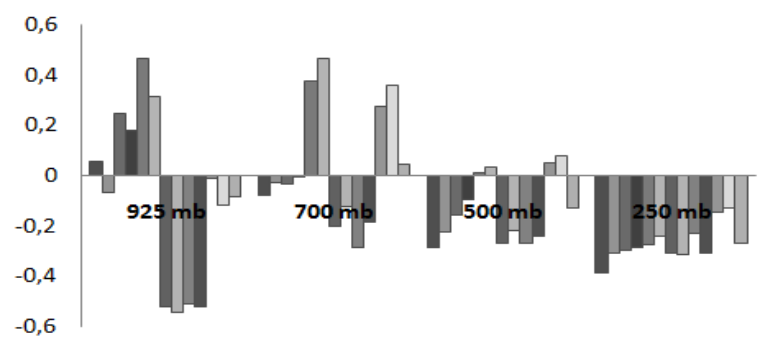

CORREL-24h

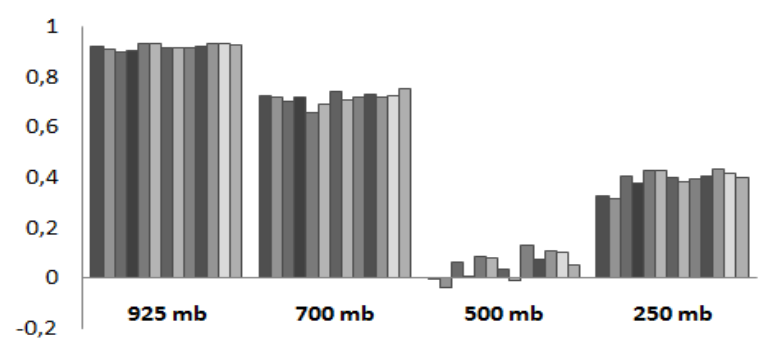

— har-kuo-mellor-24 $\square$ har-kuo-mellor-12 $\square$ har-kuo-sma-24

$\square$ har-kain-mellor-12 $\square$ chen-kuo-mellor-24 $\square$ chen-kuo-mellor-12

$\square$ chen-kain-mellor-24 $\square$ chen-kain-mellor-12 $\square$ ensemble médio
RMSE-24h

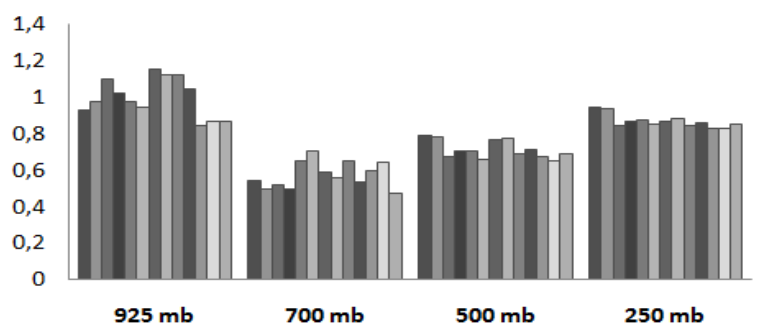

URMS-24h

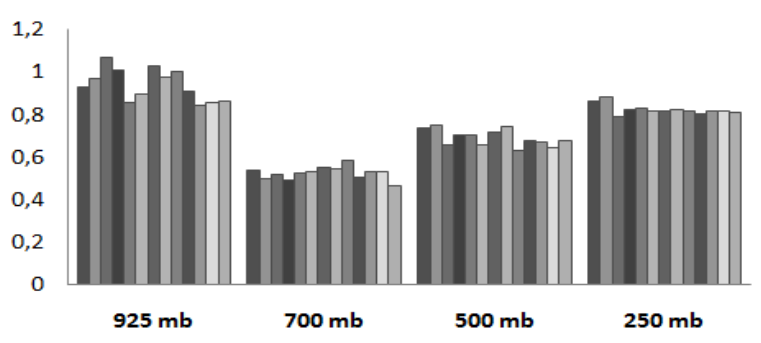

nar-kuo-sma-12 $\square$ har-kain-mellor-24

$\square$ chen-kuo-sma-24 $\square$ chen-kuo-sma-12

Figura 9 - Índices estatísticos para as previsões de 24h da temperatura para os níveis de pressão de 925 hPa, 700 hPa, 500 hPa e 250 hPa para o ENSFI.
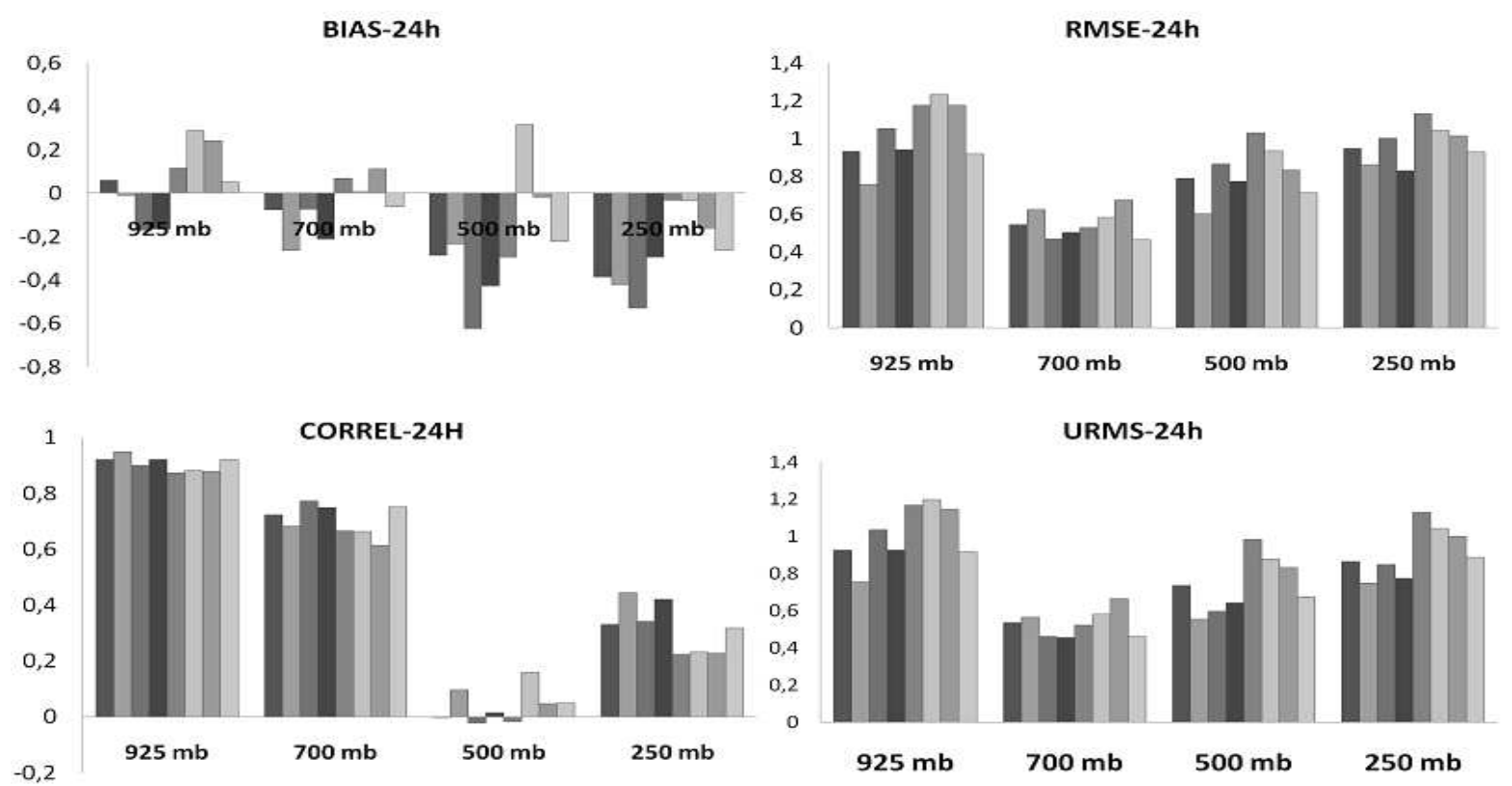

não perturbado soma pert. $24 \mathrm{~h}$ soma pert. $48 \mathrm{~h}$ soma pert. $72 \mathrm{~h}$ $\square$ subtrai pert. $24 \mathrm{~h} \square$ subtrai pert. $48 \mathrm{~h} \square$ subtrai pert. $72 \mathrm{~h} \square$ ensemble médio

Figura 10 - Índices estatísticos para as previsões de $24 \mathrm{~h}$ da temperatura para os níveis de pressão de $925 \mathrm{hPa}, 700 \mathrm{hPa}, 500 \mathrm{hPa}$ e $250 \mathrm{hPa}$ para o ENSCI-FRONT 
tendo um comportamento de subestimação, conforme indica o Viés.

\subsection{Vento em 10 metros}

Para avaliação das previsões de vento foram utilizadas 11 Plataformas de Coleta de Dados (PCDs) distribuídas por todo o estado do Ceará (Tabela 3). Nesta seção são mostradas, a título de exemplo, as tabelas e figuras que indicam o comportamento do vento observado e modelado a 10 metros de altura nas localidades de Beberibe e Granja. Para efetuar a comparação, os valores dos pontos de grade do modelo são interpolados linearmente para o ponto da referida PCD.

Nas Figuras 11 e 12 são mostradas as séries temporais de intensidade e direção do vento previstas e observadas para as localidades de Beberibe e Granja, respectivamente. As observações mostram ciclos diários bem definidos, que são bem representados pelos membros dos dois conjuntos.

Nas Tabelas 4 e 6 são mostrados os índices estatísticos dos membros do ensemble ENSFI, em relação ao vento observado para as localidades de Beberibe e Granja, respectivamente. Os melhores resultados para a intensidade do vento são obtidos para os membros utilizando a parametrização de Mellor e Yamada. Dentre estes, em relação às demais parametrizações consideradas aqui, os melhores índices são em geral obtidos com a parametrização de Harrington para a radiação. Concluindo-se, assim, que as previsões para a intensidade do vento utilizando a combinação de parametrizações de Mellor e Yamada para turbulência e Harrington para radiação apresentam resultados mais próximos aos valores observados. Os resultados para as demais PCDs utilizadas, não mostrados aqui, também indicam essa superioridade. Em relação à parametrização de convecção e

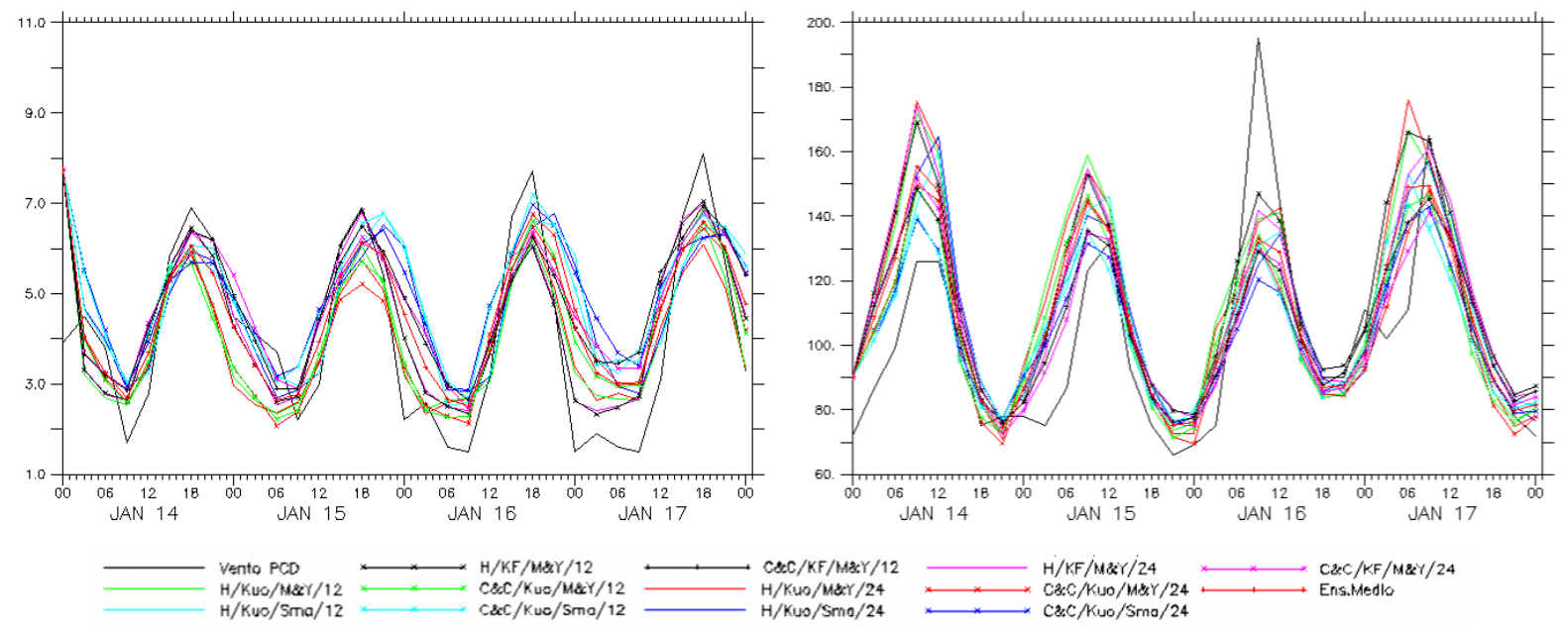

(a)

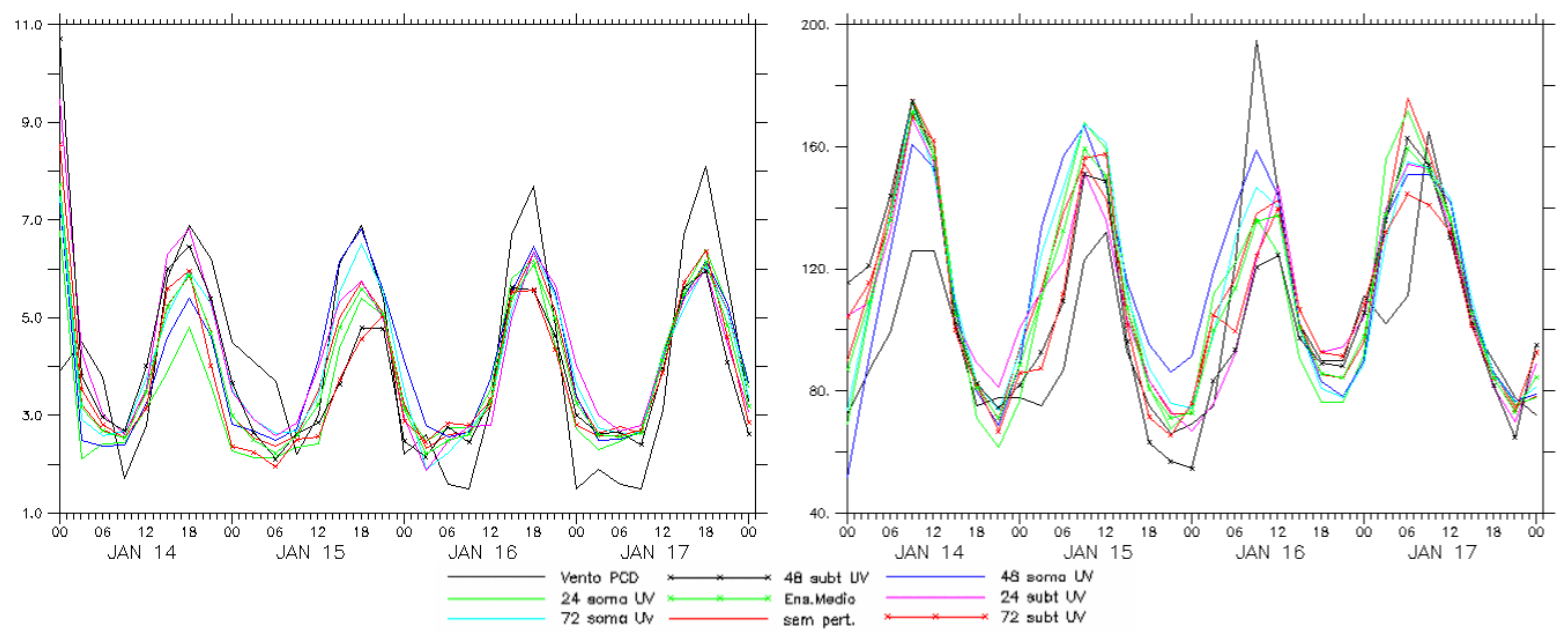

(b)

Figura 11 - Séries temporais do vento a 10m de altura registradas pela PCD de Beberibe em comparação aos membros e às médias dos ensembles: (a)ENSFI e (b)ENSCI-FRONT, sendo à esquerda a intensidade e à direita a direção do vento. 

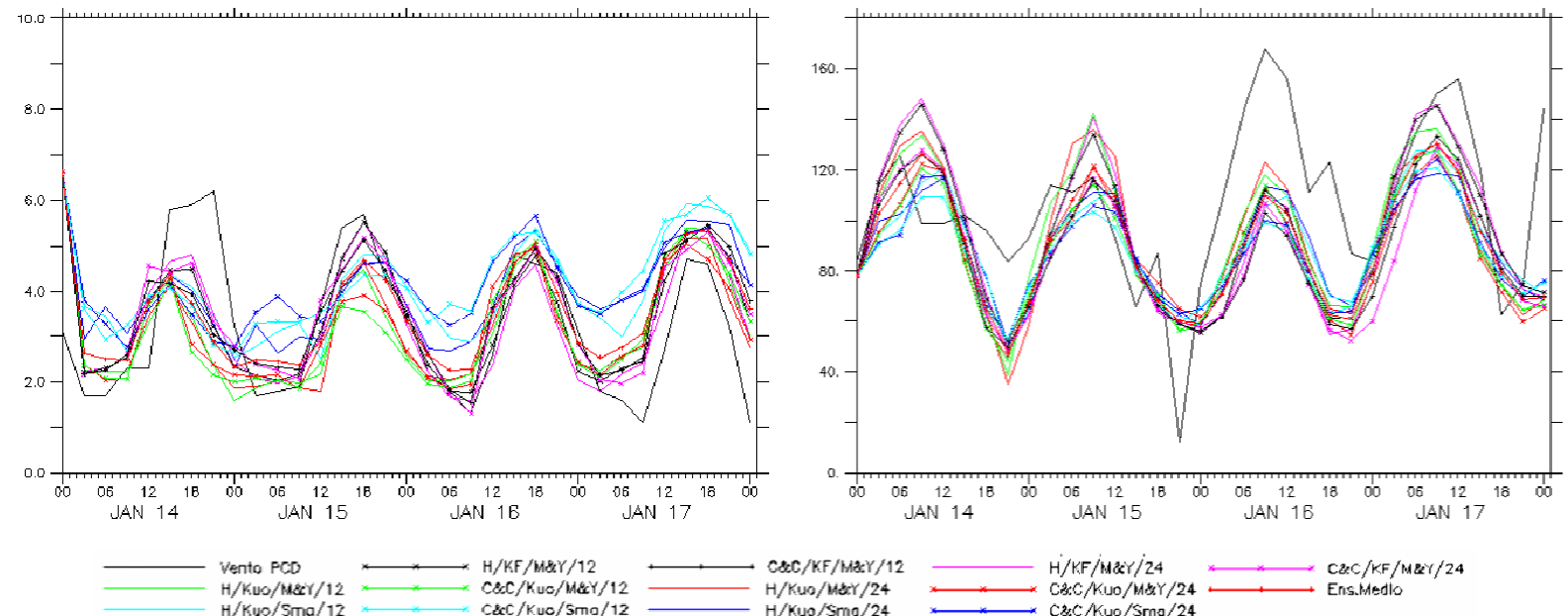

CSCC/KF/ME:Y $/ 12$

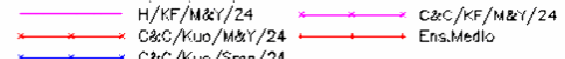

(a)
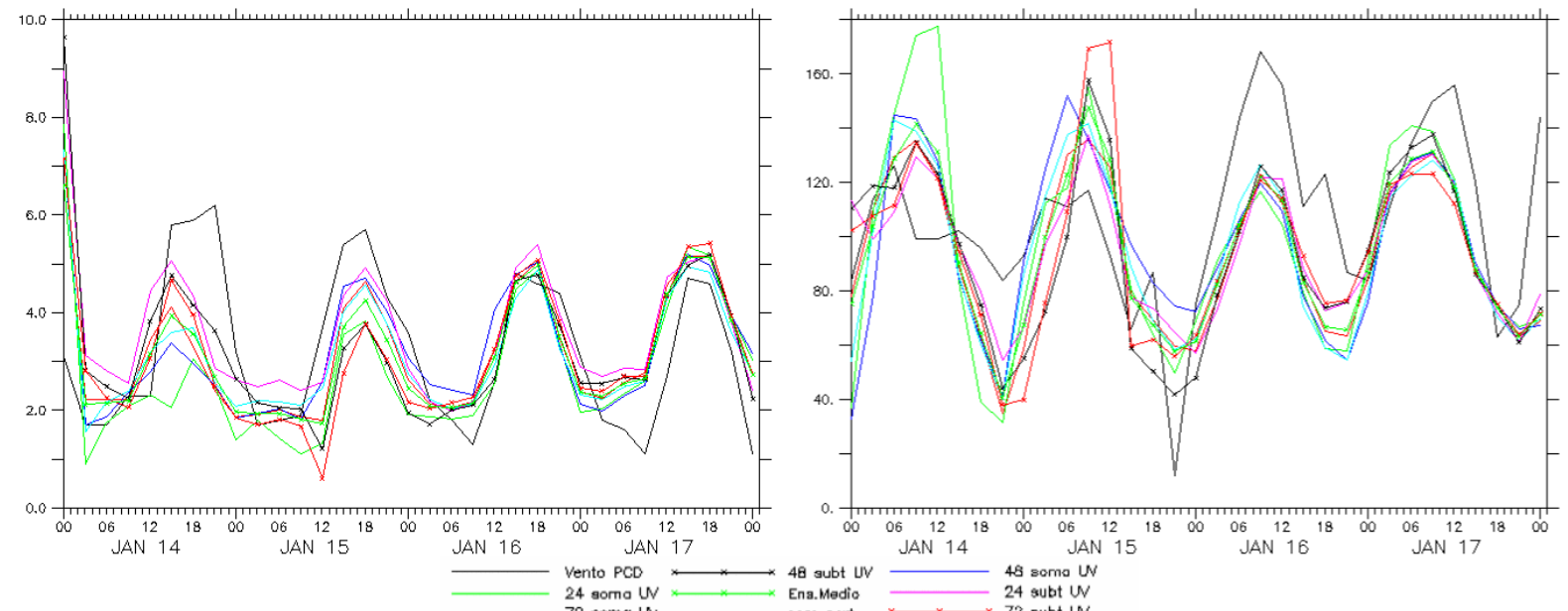

(b)

Figura 12 - Séries temporais do vento a 10m de altura registradas pela PCD de Granja em comparação aos membros e às médias dos ensembles: (a)ENSFI e (b)ENSCI-FRONT, sendo à esquerda a intensidade e à direita a direção do vento.

ao intervalo de "nudging" central, não fica evidente qual opção é responsável por melhores resultados. Para os resultados de direção do vento, também não há clareza de quais as melhores opções de parametrização. Por exemplo, as previsões utilizando a parametrização de Smagorinky para turbulência, que têm valores de índices estatísticos entre os piores para a intensidade do vento, obtêm valores entre os melhores em vários casos para a direção do vento, tanto nas PCDs mostradas nas Tabelas $4 \mathrm{e}$ 6 , quanto nas demais não mostradas aqui.

$\mathrm{Na}$ Tabela 5 são mostrados os índices estatísticos dos membros do ENSCI-FRONT em relação ao vento observado para a localidade de Beberibe. A previsão de controle não é considerada a melhor previsão nem para intensidade nem para direção do vento e, em comparação com o ensemble médio, o membro não perturbado produziu correlações piores, tanto para direção, quanto para velocidade do vento.
Na Tabela 7 são mostrados os índices estatísticos dos membros do ENSCI-FRONT em relação ao vento observado na PCD de Granja. Todos os membros representaram razoavelmente a intensidade e direção do vento nessa localidade. Nota-se, assim como em Beberibe, que a previsão de controle não é considerada a melhor previsão nem para intensidade do vento e nem para direção do vento.

\subsection{Precipitação acumulada}

Para avaliação das previsões de precipitação foram utilizadas 15 Plataformas de Coleta de Dados (PCDs) distribuídas por todo o estado do Ceará, conforme mostra a Tabela 8. Na Figura 13 são mostrados os índices estatísticos para a previsão de precipitação para o ENSFI, considerando-se todas as PCDs e as respectivas previsões para esses pontos. 
Tabela 4- Índices estatísticos da previsão da intensidade e direção do vento dos membros do ENSFI para a localidade de Beberibe em comparação aos valores registrados pela PCD dessa localidade.

\begin{tabular}{ccccc|cccc}
\hline & \multicolumn{9}{c}{ PCD de Beberibe } & \multicolumn{3}{c}{ Direção do vento a 10m } \\
Membros & VIÉS & RMSE & CORREL & URMS & VIÉS & RMSE & CORREL & URMS \\
\hline har-kuo-mellor-24 & $-0,100$ & 1,269 & 0,779 & 1,265 & 11,850 & 26,000 & 0,710 & 23,140 \\
har-kuo-mellor-12 & $-0,081$ & 1,220 & 0,798 & 1,217 & 11,960 & 25,140 & 0,722 & 22,120 \\
har-kuo-sma-24 & 0,599 & 1,619 & 0,664 & 1,504 & 7,528 & 20,670 & 0,762 & 19,250 \\
har-kuo-sma-12 & 0,686 & 1,653 & 0,665 & 1,504 & 8,018 & 20,530 & 0,767 & 18,900 \\
har-kain-mellor-24 & 0,346 & 1,167 & 0,832 & 1,114 & 11,500 & 22,400 & 0,777 & 19,230 \\
har-kain-mellor-12 & 0,315 & 1,166 & 0,829 & 1,122 & 13,570 & 23,190 & 0,787 & 18,810 \\
chen-kuo-mellor-24 & 0,161 & 1,357 & 0,742 & 1,347 & 3,161 & 19,290 & 0,765 & 19,030 \\
chen-kuo-mellor-12 & 0,153 & 1,275 & 0,777 & 1,266 & 4,170 & 19,880 & 0,753 & 19,430 \\
chen-kuo-sma-24 & 0,898 & 1,697 & 0,702 & 1,440 & 2,159 & 18,930 & 0,773 & 18,800 \\
chen-kuo-sma-12 & 0,945 & 1,647 & 0,744 & 1,348 & 2,920 & 19,470 & 0,756 & 19,250 \\
chen-kain-mellor-24 & 0,674 & 1,465 & 0,769 & 1,301 & 4,280 & 18,180 & 0,799 & 17,670 \\
chen-kain-mellor-12 & 0,680 & 1,473 & 0,764 & 1,306 & 5,566 & 18,550 & 0,799 & 17,690 \\
Ensemble médio & 0,413 & 1,308 & 0,792 & 1,241 & 7,223 & 19,980 & 0,775 & 18,630 \\
\hline
\end{tabular}

Tabela 5-Índices estatísticos da previsão da intensidade e direção do vento dos membros do ENSCI-FRONT para a localidade de Beberibe em comparação aos valores registrados pela PCD dessa localidade.

\begin{tabular}{ccccc|cccc}
\hline \hline & \multicolumn{7}{c}{ PCD de Beberibe (ENSCI-FRONT) } \\
Intensidade do Vento a 10m & \multicolumn{3}{c}{ Direção do vento a 10m } \\
Membros & VIÉS & RMSE & CORREL & URMS & VIÉS & RMSE & CORREL & URMS \\
\hline não pert. & $-0,100$ & 1,269 & 0,779 & 1,265 & 11,850 & 26,000 & 0,710 & 23,140 \\
soma pert. de 24h & $-0,411$ & 1,405 & 0,744 & 1,344 & 10,000 & 28,100 & 0,679 & 26,260 \\
soma pert. de 48h & $-0,005$ & 1,304 & 0,760 & 1,304 & 12,670 & 26,420 & 0,719 & 23,180 \\
soma pert. de 72h & $-0,021$ & 1,230 & 0,793 & 1,230 & 12,260 & 25,560 & 0,734 & 22,430 \\
subtrai pert. de 24h & 0,107 & 1,453 & 0,702 & 1,449 & 9,274 & 24,560 & 0,685 & 22,740 \\
subtrai pert. de 48h & $-0,128$ & 1,650 & 0,624 & 1,645 & 6,238 & 26,090 & 0,660 & 25,330 \\
subtrai pert. de 72h & $-0,310$ & 1,487 & 0,692 & 1,454 & 8,186 & 23,940 & 0,700 & 22,500 \\
ensemble médio & $-0,176$ & 1,277 & 0,780 & 1,264 & 10,070 & 24,090 & 0,728 & 21,880 \\
\hline
\end{tabular}

Tabela 6- Índices estatísticos da previsão da intensidade e direção do vento dos membros do ENSFI para a localidade de Granja em comparação aos valores registrados pela PCD dessa localidade.

\begin{tabular}{ccccc|cccc}
\hline \hline & \multicolumn{9}{c}{ PCD de Granja } & \multicolumn{3}{c}{ Direção do vento a 10m } \\
Membros & VIÉS & RMSE & CORREL & URMS & VIÉS & RMSE & CORREL & URMS \\
\hline har-kuo-mellor-24 & $-0,019$ & 1,367 & 0,532 & 1,367 & $-12,990$ & 31,000 & 0,561 & 28,150 \\
har-kuo-mellor-12 & $-0,008$ & 1,381 & 0,526 & 1,381 & $-12,340$ & 29,690 & 0,588 & 27,000 \\
har-kuo-sma-24 & 0,752 & 1,626 & 0,425 & 1,441 & $-16,110$ & 30,130 & 0,589 & 25,460 \\
har-kuo-sma-12 & 0,844 & 1,685 & 0,424 & 1,459 & $-15,340$ & 30,240 & 0,567 & 26,060 \\
har-kain-mellor-24 & 0,135 & 1,099 & 0,721 & 1,090 & $-11,400$ & 33,370 & 0,489 & 31,360 \\
har-kain-mellor-12 & 0,222 & 1,171 & 0,685 & 1,150 & $-11,190$ & 31,610 & 0,521 & 29,560 \\
chen-kuo-mellor-24 & 0,000 & 1,423 & 0,480 & 1,423 & $-19,280$ & 33,370 & 0,533 & 27,230 \\
chen-kuo-mellor-12 & $-0,030$ & 1,528 & 0,413 & 1,528 & $-19,300$ & 32,330 & 0,581 & 25,930 \\
chen-kuo-sma-24 & 0,889 & 1,783 & 0,280 & 1,545 & $-18,740$ & 32,570 & 0,534 & 26,640 \\
chen-kuo-sma-12 & 0,965 & 1,897 & 0,221 & 1,634 & $-19,820$ & 32,420 & 0,579 & 25,660 \\
chen-kain-mellor-24 & 0,319 & 1,191 & 0,683 & 1,148 & $-20,050$ & 34,800 & 0,505 & 28,440 \\
chen-kain-mellor-12 & 0,332 & 1,302 & 0,610 & 1,259 & $-18,850$ & 34,000 & 0,508 & 28,300 \\
ensemble médio & 0,342 & 1,364 & 0,546 & 1,320 & $-16,290$ & 31,320 & 0,557 & 26,750 \\
\hline
\end{tabular}


Tabela 7- Índices estatísticos da previsão da intensidade e direção do vento dos membros do ENSCI-FRONT para a localidade de Granja em comparação aos valores registrados pela PCD dessa localidade.

\begin{tabular}{ccccc|cccc}
\hline & \multicolumn{9}{c}{$\begin{array}{c}\text { PCD de Granja (ENSCI-FRONT) } \\
\text { Vento a 10m }\end{array}$} & \multicolumn{3}{c}{ Direção do vento a 10m } \\
Membros & VIÉS & RMSE & CORREL & URMS & VIÉS & RMSE & CORREL & URMS \\
\hline não pert. & $-0,019$ & 1,367 & 0,532 & 1,367 & $-12,990$ & 31,000 & 0,561 & 28,150 \\
soma pert. 24h & $-0,396$ & 1,691 & 0,403 & 1,644 & $-9,847$ & 37,810 & 0,459 & 36,510 \\
soma pert. 48h & 0,018 & 1,430 & 0,504 & 1,430 & $-10,800$ & 34,350 & 0,452 & 32,610 \\
soma pert. 72h & $-0,046$ & 1,363 & 0,531 & 1,362 & $-12,010$ & 31,280 & 0,562 & 28,890 \\
subtrai pert. 24h & 0,410 & 1,512 & 0,515 & 1,456 & $-11,350$ & 28,320 & 0,590 & 25,950 \\
subtrai pert. 48h & 0,075 & 1,634 & 0,438 & 1,633 & $-12,300$ & 31,190 & 0,581 & 28,670 \\
subtrai pert. 72h & $-0,080$ & 1,526 & 0,453 & 1,524 & $-11,580$ & 34,620 & 0,464 & 32,630 \\
ensemble médio & $-0,080$ & 1,346 & 0,543 & 1,344 & $-11,550$ & 30,670 & 0,555 & 28,410 \\
\hline
\end{tabular}

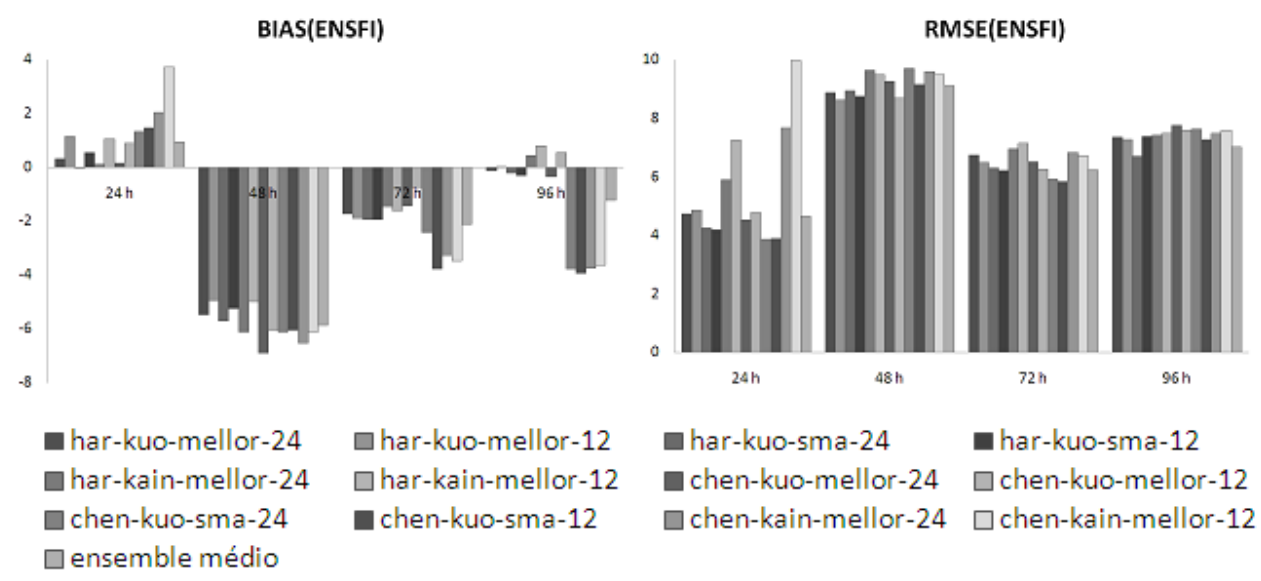

Figura 13 -Índices estatísticos para precipitação para as previsões de 24 h, 48 h, 72 h e 96 h dos membros doENSFI

Tabela 8-PCDs usadas para avaliação da precipitação prevista.

\begin{tabular}{ccc} 
PCD & Latitude & Longitude \\
\hline Acopiara & $06^{\circ} 05^{\prime} 53.6^{\prime \prime}$ & $39^{\circ} 12^{\prime} 36.5^{\prime \prime}$ \\
Aratuba & $04^{\circ} 23^{\prime} 20,9^{\prime \prime}$ & $39^{\circ} 02^{\prime} 27,5^{\prime \prime}$ \\
Barroquinha & $02^{\circ} 55^{\prime} 48.2^{\prime \prime}$ & $41^{\circ} 07^{\prime} 07.3^{\prime \prime}$ \\
Ibaretama & $0^{\circ} 49^{\prime} 36,8^{\prime \prime}$ & $38^{\circ} 49^{\prime} 51,0^{\prime \prime}$ \\
Ipu & $0^{\circ} 25^{\prime} 36,6^{\prime \prime}$ & $40^{\circ} 42^{\prime} 51,0^{\prime \prime}$ \\
Itapipoca - Quandu & $03^{\circ} 34^{\prime} 24.1^{\prime \prime}$ & $39^{\circ} 37^{\prime} 40.0^{\prime \prime}$ \\
Itaiçaba & $04^{\circ} 44^{\prime} 02.6^{\prime \prime}$ & $37^{\circ} 50^{\prime} 52.6^{\prime \prime}$ \\
Maranguape & $03^{\circ} 59^{\prime} 06,1^{\prime \prime}$ & $38^{\circ} 47^{\prime} 17,5^{\prime \prime}$ \\
Meruoca & $03^{\circ} 32^{\prime} 32,2^{\prime \prime}$ & $40^{\circ} 29^{\prime} 59,9^{\prime \prime}$ \\
Pacajus & $04^{\circ} 11^{\prime} 24,0^{\prime \prime}$ & $38^{\circ} 29^{\prime} 54,2^{\prime \prime}$ \\
Pereiro & $06^{\circ} 02^{\prime} 23.5^{\prime \prime}$ & $38^{\circ} 28^{\prime} 02.0^{\prime \prime}$ \\
Santana do Cariri & $07^{\circ} 09^{\prime} 06.5^{\prime \prime}$ & $39^{\circ} 45^{\prime} 52.1^{\prime \prime}$ \\
Senador Pompeu & $05^{\circ} 34^{\prime} 50.9^{\prime \prime}$ & $39^{\circ} 23^{\prime} 56.7^{\prime \prime}$ \\
Tabuleiro do Norte & $05^{\circ} 29^{\prime} 25,5^{\prime \prime}$ & $38^{\circ} 01^{\prime} 28,6^{\prime \prime}$ \\
Tamboril & $04^{\circ} 49^{\prime} 18.8^{\prime \prime}$ & $40^{\circ} 21^{\prime} 40.8^{\prime \prime}$ \\
\hline
\end{tabular}

No ENSFI, os membros tendem a subestimar a precipitação das PCDs para prazos de previsão a partir de 48 horas. O conjunto apresenta RMSE relativamente alto, principalmente para previsão de 48 horas.

Comparando-se o ensemble médio e membro de controle, estes apresentam índices muito semelhantes entre si, não sendo possível identificar uma superioridade marcante entre eles.

Na Figura 14 é feita uma comparação individual, entre os valores dados para a precipitação acumulada a cada 24 horas pela previsão de controle e pela média das previsões com os métodos ENSCI-FRONT e ENSFI, tendo por base os resultados do Viés nas 15 PCDs espalhadas pelo Ceará (conforme mostra a Tabela 8). Ao comparar-se os valores dados pelas previsões do membro de controle e do ensemble médio do ENSFI e ENSCIFRONT em relação a qual se encontra mais próximo do valor observado para precipitação acumulada em 24 horas em cada PCD, nota-se que no primeiro dia de simulação a previsão determinística mostra-se claramente superior, mas já no segundo dia de simulação essa diferença torna-se menor. Para o terceiro e o quarto dia, é o ensemble médio que apresenta resultados melhores, tanto para o ENSFI, quanto para o ENSCI-FRONT. 


\section{CONCLUSÕES}

As previsões geradas pelo ENSCI apresentam um espalhamento muito pequeno sobre o domínio de grade utilizado, principalmente ao se considerar períodos mais afastados das condições iniciais. Isso ocorre porque todos os membros do modelo regional são forçados pelas mesmas condições de grande escala do MCGA. Assim, a variabilidade introduzida nas condições iniciais é rapidamente transportada para fora do domínio do modelo regional, sendo essa informação substituída gradualmente, através do nudging, por uma outra, que é idêntica em todos os membros. Conclui-se, portanto, que não é suficiente perturbar apenas as condições iniciais quando se trata de um ensemble de simulações de um modelo regional, sendo necessário adicionar o termo de perturbação ao longo de todo o período de previsão

Quando se comparam as diversas combinações de parametrizações do ENSFI, não é possível definir qual a melhor opção considerando todos os níveis de pressão e todas as variáveis. Existem, porém, algumas tendências que são relevantes nas previsões que precisam ser destacadas e, dependendo do propósito do previsor, pode haver uma escolha que se sobressaia diante das outras. Dentre os membros desse conjunto, aqueles que combinam as parametrizações de KainFritsch e Smagorinsky não completaram as simulações, fato acarretado possivelmente por instabilidades numéricas geradas pelo uso conjunto destas duas parametrizações.

Quanto à pressão na superfície, há uma melhor representação pelos membros que usam a parametrização de convecção de Kuo nas primeiras horas de simulação, em relação aos membros correspondentes que usam a parametrização de Kain-Fritsch para convecção, porém esse comportamento inverte-se nas previsões de $72 \mathrm{~h}$ e $96 \mathrm{~h}$, indicando certa instabilidade do esquema de Kuo.

Quanto à temperatura, embora haja um bom desempenho da previsão tradicional próximo à superfície, há dificuldade em prever essa variável nos níveis de pressão de 250 e 500
hPa. Os métodos de ensemble utilizados não resultam em melhoras significativas para essas previsões, o que indica o problema em prever a evolução da estrutura termodinâmica da atmosfera sobre o Nordeste do Brasil em 500 e $250 \mathrm{hPa}$. Isso é importante pois, especialmente em janeiro, sabe-se que a circulação atmosférica em altos níveis é muito relevante para a previsão de tempo sobre essa região, já que as condições sinópticas associadas à ocorrência de precipitação nesse período comumente se relacionam ao posicionamento do cavado ou a formação de vórtices ciclônicos em altos níveis(Alburquerque et al., 2009).

Há uma boa representação do vento observado pelas duas técnicas de previsão por conjuntos, principalmente para sua intensidade, o que sugere a aplicabilidade da metodologia de ensemble para a previsão do vento. Os membros que combinam as parametrizações de Mellor e Yamada para turbulência e Harrington para radiação produzem melhores previsões para a intensidade do vento. Para a direção do vento, e para as demais escolhas de diferentes configurações utilizadas, os resultados obtidos não mostram clareza quanto a quais seriam as melhores opções.

Considerando-se a precipitação sobre o estado do Ceará, as médias das previsões geradas, tanto pelo ENSFI, quanto pelo ENSCI-FRONT, mostram-se superiores à previsão de controle para prazos de previsão maiores que 48 horas na maioria das PCDs. Isso sugere que o ensemble médio pode melhorar o prazo de validade das previsões e torná-las mais confiáveis.

Em relação à escala de tempo de "nudging", os membros que usam parâmetros de "nudging" diferentes apresentam previsões bem semelhantes para praticamente todas as variáveis em todos os níveis de pressão, portanto, para o tempo de simulação utilizado neste trabalho, não se justifica a variação desse parâmetro no conjunto.

No caso do ensemble ENSCI-FRONT, mesmo quando o ensemble médio não produz melhor previsão que o controle, é comum um dos membros perturbados apresentar uma previsão mais coerente.
Controle x EM(ENSCI-FRONT)

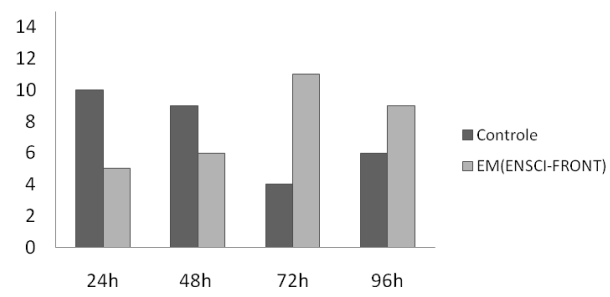

(a)
Controle x EM(ENSFI)

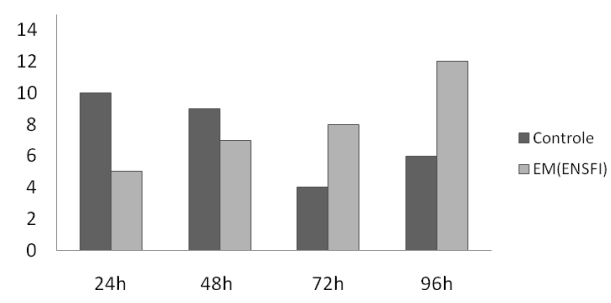

(b)

Figura 14 -Comparação entre a previsão dada pelo membro de controle e pela média dos membros em relação aos valores observados de precipitação para os métodos: (a) ENSCI-FRONT; (b) ENSFI. Número de PCDs onde cada técnica obteve o menor viés. 
Admitindo-se o comportamento caótico da atmosfera e a incapacidade de eliminar as incertezas das condições iniciais e de descrever completamente os processos físicos e dinâmicos da atmosfera, a previsão de tempo por conjuntos surge como uma resposta para lidar com essas incertezas e melhorar o índice de acerto da previsão determinística, com a possibilidade de transformar a previsão do tempo num evento probabilístico. Diante disso, a ferramenta exposta nesse trabalho é uma técnica importante no desenvolvimento da previsão do tempo.

Neste trabalho foram apresentados resultados de vários experimentos, onde diversas combinações de parametrizações físicas são testadas em um sistema de previsão por conjunto sobre a região Nordeste do Brasil usando o RAMS. No entanto, foi utilizado apenas um intervalo de integração e avaliação da metodologia. Essa limitação indica que novos testes precisam ser feitos, para identificar se a atuação de outros sistemas meteorológicos não alterariam os resultados obtidos neste trabalho. Novos testes deverão ser executados para fornecer um conjunto de informações mais detalhado e melhorar entendimento em relação à confiabilidade, à sensibilidade e às limitações do sistema de previsão exposto.

\section{REFERÊNCIAS}

ALBURQUERQUE, I. F. et al.Tempo e Clima no Brasil. São Paulo. Oficina de Textos p. 280, 2009.

BUIZZA, R. ; PALMER, T.N.. The singular-vector structure of the atmospheric general circulation. Journal of the Atmospheric Sciences, v. 52, p. 1434-1456,1995.

BUSTAMANTE, J. ; CHOU, S. C. Estudo da previsibilidade de eventos extremos sobre a serra do mar utilizando ensemble de curto prazo. In: XIV Congresso Brasileiro de Meteorologia, 2006, Florianópolis. XIV Congresso Brasileiro de Meteorologia, 2006.

CHEN, C. ; COTTON W. R. Numerical experiments with a onedimensional higher order turbulence model: Simulation of the Wangara day 33 case. Boundary Layer Meteorology, v. 25 , p. $375-404,1983$.

COTTON,C. et al. RAMS 2001: Current status and future directions. Meteorology and Atmospheric Physics, v. 82, n. 1, p. 5-29, 2003.

COUTINHO, M.M. Previsão por conjuntos utilizando perturbações baseadas em componentes principais. 1999. 136f. Dissertação (Mestrado em Meteorologia), Instituto Nacional de Pesquisas Espaciais - INPE, São José dos Campos, SP, 1999.

DALEY, R.;MAYER, T. Estimates of global analysis error from the global weather experiment observational network. Monthly Weather Review, v. 114, p. 1642-1653, 1986
DE MARIA, P.H.S. et al. Modelagem numérica em alta resolução para previsão de geração de energia eólicano Ceará. Revista Brasileira de Meteorologia, v. 23, n. 4, p. 477-489, 2008.

HARRINGTON, J. The effects of radiative and microphysical processes on simulated warm and transition season arctic stratus. PhD. Colorado State University, 1997.

HOFFMAN, R. N.; KALNAY, E. Lagged Average Forecasting, an Alternative to Monte Carlo Forecasting. Tellus, v. 35A, n. 2, p. 100-118, 1983.

HOU, E. D. K. et al. Objetive Verification of the SAMEX' 98 Ensemble Forecast. Monthly Weather Review, v. 129, p. 73-91, 2001.

KAIN, S. J. The Kain-Fritsch Convective Parameterization: An Update. Journal of Applied Meteorology, v. 43, p.170181, 2002.

KRISHNAMURTI, T.N.; ZHANG, Z. A perturbation method for hurricane ensemble predictions. Monthly Weather Review, v. 127, n. 4, p. 447-469, 1999.

LEITH, C. E Theoretical Skill of Monte Carlo Forescast. Monthly Weather Review,v.102, n.6, p.409-418,1974.

LORENZ, E. N. Deterministic non-periodic flow. Journal of the Atmospheric Sciences, v. 20, n. 2, p. 130-141, 1963.

LORENZ, E. N. A study of the predictability of a 28 -Variable Atmospheric Model. Tellus, v. 17, n. 3, p. 321-333, 1965.

MELLOR, G.; YAMADA, T. A hierarchy of turbulence closure models for atmospheric boundary layers. Journal of the Atmospheric Sciences, v. 31, p. 1791-1806, 1974.

SILVEIRA, C. S. Previsão de Tempo por Ensemble Regional para o Nordeste Brasileiro. 2008. 202f. Dissertação (Mestrado Acadêmico em Ciências Físicas Aplicadas) Universidade Estadual do Ceará, Fortaleza, 2008.

SILVEIRA,C.S. et al. Verificação das previsões de tempo para precipitação usando ensemble regional para o estado do Ceará em 2009. Revista Brasileira de Meteorologia, v. 26, n. 4, 609-618, 2011.

SMAGORINSKY, J. General circulation experiments with the primitive equations. Monthly Weather Review, v. 91, n. 3, p. 99-164, 1963.

STENSURD,D.J. et al. Ensemble Forcasting of mesoscale convective system. Preprints, 12th conf.numerical weather prediction, Phoenix, AZ, American Meteorological Society, p. 265-268, 1998.

TREMBACK, C. Numerical Simulation of a Mesoscale Convective Complex: Model Development and Numerical Results. 289f. 1990. Tese (Department of Atmospheric Science) - Colorado State University, 1990

TOTH, Z.; KALNAY, E. Ensemble Forecasting at NMC: The Generation of Perturbations. Bulletin of the American Meteorological Society, v.74, (12), p 2317-2339, Dec. 1993. WILKS, D.S Statistical Methods in the Atmospheric Science. San Diego: Academic Press, 467 p,1995. 